\title{
The stochastic interactions between predator and prey under Markovian switching: competitive interaction between multiple prey
}

\author{
Yanqing Li, Long Zhang* \\ College of Mathematics and System Sciences, Xinjiang University, Urumqi 830046, P. R. China.
}

Communicated by A. Atangana

\begin{abstract}
In this paper, a class of predator-prey model with prey competition is proposed, in which the interactions of predation between predator and prey are randomised and subsequently evaluated under Markovian switching. By constructing appropriate Lyapunov functions and applying various analytical methods, sufficient conditions for the existence of unique global positive solution, stochastic permanence and mean extinction are established. In the permanence case, we also estimate the superior and inferior limits of the sample path in a time-averaged Markov decision. We conclude that the interactions between predator and two prey, two competitive prey themselves and the dynamical properties of switching subsystems are not only dependent on subsystem coefficients but also on the transition probability of the Markov chain (switching from one state to another). Specific examples and numerical simulations are provided to demonstrate our theoretical results. (C)2017 All rights reserved.
\end{abstract}

Keywords: Random selection, competition between prey, Markovian switching, stochastic permanence, extinct in mean. 2010 MSC: 92D25, 34D20, 34D10.

\section{Introduction}

The dynamic behaviour between predator and prey has long been and will continue to be one of the dominant themes in both ecology and mathematical ecology due to its universal existence and importance [4]. During the past few decades, predator-prey models have been extensively investigated [1-3, 9, 10, 18, $25,35,36]$, including the review of classical work and monographs, i.e., Gauss-types [18], Leslie-Gower models [10], ratio-dependent predator-prey systems [1, 25], Holling types [9, 35], Beddington-DeAngelis functional response models $[3,36]$, etc. The classical Lotka-Volterra predator-prey model is

$$
\left\{\begin{array}{l}
\frac{d x(t)}{d t}=x(t)(a-b y(t)), \\
\frac{d y(t)}{d t}=y(t)(-c+d x(t)),
\end{array}\right.
$$

where $x$ and $y$ represent the prey and predator population density, respectively; $a$ is the intrinsic growth rate of population $x ; b$ is the capturing rate of predator $y ; c$ and $d$ are the interspecific competition

\footnotetext{
*Corresponding author

Email addresses: 1548510640@qq.com (Yanqing Li), Longzhang_xj@sohu.com (Long Zhang)
} 
coefficients and rate of nutrients conversion of the population $y$, respectively. This is a deterministic predator-prey model, i.e., the parameters are all deterministic irrespective of environmental fluctuations. Many significant studies concerned with this model have been researched $[7,8,27]$. However, due to the complexity of the process in nature, population systems are often subject to environmental noise, which is an important component in the real ecosystem. A system with such random perturbations tends to be suitably modelled by stochastic differential equations [12, 17, 37]. Nisbet and Gurney [22] demonstrated that stochastic differential equations models play a significant role in the analysis of various dynamic systems, because they can provide an additional degree of realism compared to their deterministic counterpart. May [20] also noted that due to environmental fluctuation, birth rates, carrying capacity, competition coefficients and other parameters involved in a population model system exhibit random fluctuation to a greater or lesser extent. Therefore, many authors introduced stochastic perturbations into the deterministic models [16, 19, 29, 33]. Takeuchi et al. [28] considered the following predator-prey model with telegraph noise based on model (1.1):

$$
\left\{\begin{array}{l}
\frac{d x(t)}{d t}=x(t)(a(r(t))-b(r(t)) y(t)), \\
\frac{d y(t)}{d t}=y(t)(-c(r(t))+d(r(t)) x(t)),
\end{array}\right.
$$

where $r(t)$ is a right-continuous Markov chain on the state space $S=\{1,2\}$. The authors stated that telegraph noise can be expressed as a switch between two environmental regimes, which are differentiated by elements such as nutrition or rain-falls. Telegraph noise is memoryless, and the waiting time for the next change has an exponential distribution. Therefore, the population model (1.2) under regime switching can be described by two deterministic systems with different parameters. Authors revealed a very interesting and surprising result achieved via analytical methods: under the influence of telegraph noise, if two equilibrium states of the subsystems are different, then all positive trajectories of the system will be away from any compact set of $R_{+}^{2}$ with probability one. When the two equilibrium states coincide with each other, the trajectory either exists from a random compact set of $R_{+}^{2}$ or converges to the equilibrium state. In fact, two equilibrium states often do not coincide with each other. Takeuchi et al. [28] discovered that the stochastic species system is neither permanent nor dissipative (see, e.g., [5]). This is an important result as it reveals a significant effect on the species system, i.e., both its subsystems evolved periodically, but the switching made them neither permanent nor dissipative.

On the other hand, Hutchinson [11] stated that differential predation on competitive prey species may theoretically permit some diversification of the prey population. To evaluate the effect of predation on species diversity for competing species, Parrish et al. [23] proposed a three-species model that described competition between prey species 1 and 2, where predator species 3 preyed on both prey species 1 and 2 as follows:

$$
\left\{\begin{array}{l}
\frac{d x_{1}(t)}{d t}=x_{1}(t)\left[\varepsilon_{1}-\alpha_{11} x_{1}(t)-\alpha_{12} x_{2}(t)-\alpha_{13} y(t)\right], \\
\frac{d x_{2}(t)}{d t}=x_{2}(t)\left[\varepsilon_{2}-\alpha_{22} x_{2}(t)-\alpha_{21} x_{1}(t)-\alpha_{23} y(t)\right], \\
\frac{d y(t)}{d t}=y(t)\left[\varepsilon_{3}+\alpha_{31} x_{1}(t)+\alpha_{32} x_{2}(t)\right]
\end{array}\right.
$$

where $\varepsilon_{i}(i=1,2,3)$ are the intrinsic growth rates of species $x_{i}(i=1,2)$ and the predator species $y$; $\alpha_{11}, \alpha_{12}, \alpha_{21}, \alpha_{22}$ represent the intra- and inter-specific competitive coefficients of two prey; the coefficients $\alpha_{13}, \alpha_{23}$ represent the capturing rate of predator; and $\alpha_{31}, \alpha_{32}$ represent the rate of conversion of nutrients. This is a deterministic system, the interactions between predator $y$ and prey $x_{i}(i=1,2)$, the competitive intensity between multiple prey, are fixed, and unalterable. However, in the realistic setting, because of intrinsic physiology of environment or human intervention over time, the predation randomly occurs and competitive outcome changes. For example, when resource is rich, predators (such as a carnivore) tend to specialise in eating prey types that are easy to catch and subdue, and avoid prey types 
that are distasteful or noxious in some way. Meanwhile, when living conditions become restricted, i.e., these "suitable prey" number are in the low phase of their cycle, predators switch to "alternate prey" [24]. In this means, the competitive results on two prey may also change [21]. Following these situations, the interactions between predator and prey and the force of competition on two prey can be determined stochastically by randomly switching. And this action is memoryless and restricted to an exponential distribution, i.e., a Markov switching system [13-15, 30-32, 34].

Based on the arguments above, in this paper, we research a class of predator-prey models with stochastic interactions of predator and prey under Markovian switching, including the competition between two prey species:

$$
\left\{\begin{array}{l}
\frac{d x_{1}(t)}{d t}=x_{1}(t)\left[a_{1}(r(t))-b_{1}(r(t)) x_{1}(t)-d_{1}(r(t)) y-g_{1}(r(t)) x_{2}(t)\right] \\
\frac{d x_{2}(t)}{d t}=x_{2}(t)\left[a_{2}(r(t))-b_{2}(r(t)) x_{2}(t)-d_{2}(r(t)) y(t)-g_{2}(r(t)) x_{1}(t)\right] \\
\frac{d y(t)}{d t}=y(t)\left[a_{3}(r(t))-b_{3}(r(t)) y(t)+e_{1}(r(t)) x_{1}(t)+e_{2}(r(t)) x_{2}(t)\right]
\end{array}\right.
$$

where we assume that the vector $x=\left(x_{1}, x_{2}, y\right)^{\top}$ represents the population density of prey species $x_{i}(i=$ $1,2)$ and predator species $y ; r(t)$ is a stochastic process taking values in a finite state space $\tilde{S}=\{0,1,2,3\}$; $a_{i}(r(t))(i=1,2,3)$ denotes the intrinsic growth rate of predator and prey under state $r(t) ; b_{i}(r(t))$ $(i=1,2,3)$ represents the intraspecific competitive coefficient of the multiple prey species $x_{i}(i=1,2)$ and predator under state $r(t) ; d_{i}(r(t))(i=1,2)$ is the capturing rate of the predator at time interval under state $r(t) ; e_{i}(r(t))(i=1,2)$ is the rate of conversion of nutrients of the predator under state $r(t)$. This system may be characterised by the following set of cases.

Case 1. $r(t)=0$. In this case, the predator species $y$ has other food resource and does not prey on species $x_{1}$ and $x_{2}$, but the prey species $x_{i}(i=1,2)$ compete with each other. Therefore, $e_{i}(0)=d_{i}(0)=0(i=1,2)$ while other parameters are nonzero constants.

Case 2. $r(t)=1$. In this case, the predator $y$ captures prey $x_{1}$ in time, meanwhile, there exists competition between the prey $x_{1}$ and $x_{2}$, i.e., $e_{2}(1)=d_{2}(1)=0$, while other parameters are nonzero constants.

Case 3. $r(t)=2$. In this case, which is like Case 2 , only the prey $x_{2}$ can be caught by predator $y$. Therefore, system (1.3) becomes another subsystem in which the predator $y$ captures prey $x_{2}$ and the remaining prey $x_{1}$ and $x_{2}$ compete with each other.

Case 4. $r(t)=3$. In this case, for example, when winter comes, some species vegetarians or birds species will migrate to resource-rich food habitats (or warm place) in search for a better place to breed and survive, predator $y$ have no choice but to catch species $x_{1}$ and $x_{2}$. Thus the interaction among the three species becomes a two-prey-one-predator system where preys compete with each other. This implies that all parameters in system (1.3) are nonzero constants.

Therefore, system (1.3) can be regarded as an interaction between four deterministic subsystems. The law of the Markov chain switching is applied in these situations. In the real ecosystem, owing to natural enemies, competition, seasonal alternatives or deterioration of patches of the environment, species movement behaviour is common. Therefore, we conclude that the system (1.3) is reasonable.

In this paper, we investigate the dynamic behaviour of system (1.3) (i.e., the existence of the unique global stochastic positive solution, stochastic permanence, extinction, and path-wise estimation) and explore the influence of Markvian switching on the population dynamic of multiple species (1.3). The paper is organised as follows. In Section 2, preliminaries are introduced. In Section 3, we study the existence and uniqueness of the globally positive solution of system (1.3). Sufficient conditions for the extinction and stochastic permanence are established in Section 4. Path-wise estimation is discussed in Section 5. In Section 6, we present specific numerical examples to demonstrate the theoretical results. A discussion is provided in Section 7. 


\section{Preliminaries}

Let $\left(\Omega, \mathcal{F},\left\{\mathcal{F}_{t}\right\}_{t} \geqslant 0, P\right)$ be a complete probability space with a filtration $\left\{\mathcal{F}_{t}\right\}_{t \geqslant 0}$ satisfying the usual conditions (i.e., it is right-continuous and $\mathcal{F}_{0}$ contains all P-null set). Let $r(t)$ be a right-continuous Markov chain on the probability space, and taking values in a finite state $\tilde{S}=\{0,1,2,3\}$ with the generator $\Pi=\left(\pi_{\mathrm{IJ}}\right)_{4 \times 4}$ given by

$$
\mathrm{P}\{\mathrm{r}(\mathrm{t}+\triangle)=\mathrm{J} \mid \mathrm{r}(\mathrm{t})=\mathrm{I}\}= \begin{cases}\pi_{\mathrm{IJ}} \triangle+\mathrm{o}(\triangle), & \mathrm{I} \neq \mathrm{J}, \\ 1+\pi_{\mathrm{IJ}} \triangle+\mathrm{o}(\triangle), & \mathrm{I}=\mathrm{J},\end{cases}
$$

where $I, J \in \tilde{S}, \triangle>0, \lim _{\triangle \rightarrow 0} \frac{o(\triangle)}{\triangle}=0$. Here, $\pi_{I J}$ is the transition rate from I to J and $\pi_{I J} \geqslant 0(I, J \in \tilde{S}, I \neq J)$, while

$$
\pi_{\mathrm{II}}=-\sum_{\mathrm{J}=1, \mathrm{~J} \neq \mathrm{I}}^{4} \pi_{\mathrm{IJ}}
$$

For convenience and simplicity in the following discussion, for any constant sequence

$$
\left\{c_{i j}(I)\right\} \quad(1 \leqslant i, j \leqslant 3, \quad I \in \tilde{S}),
$$

define

$$
\begin{array}{ll}
\check{\mathbf{c}}=\max _{1 \leqslant i, j \leqslant 3, I \in \tilde{S}} c_{i j}(I), \quad \check{c}(I)=\max _{1 \leqslant i, j \leqslant 3} c_{i j}(I), \\
\hat{\mathbf{c}}=\min _{1 \leqslant i, j \leqslant 3, I \in \tilde{S}} c_{i j}(I), \quad \hat{c}(I)=\min _{1 \leqslant i, j \leqslant 3} c_{i j}(I) .
\end{array}
$$

Moreover, we rewrite (1.3) to

$$
\left\{\begin{array}{l}
\dot{x}(t)=\operatorname{diag}\left(x_{1}(t), x_{2}(t), y(t)\right)[a(r(t))+A(r(t)) x(t)], \\
x\left(\tau_{0}^{+}\right)=x_{0}>0, r\left(\tau_{0}^{+}\right)=r_{0} \in \tilde{S},
\end{array}\right.
$$

where $x=\left(x_{1}(t), x_{2}(t), y(t)\right)^{\top} \in R^{3}, a(I)=\left(a_{1}(I), a_{2}(I), a_{3}(I)\right)^{\top} \in R^{3}$ represents the intrinsic growth rate of the species for a fixed $I \in \tilde{S}$, and

$$
A=(A(r(t)))_{3 \times 3}=\left(\begin{array}{ccc}
-b_{1}(r(t)) & -g_{1}(r(t)) & -d_{1}(r(t)) \\
-g_{2}(r(t)) & -b_{2}(r(t)) & -d_{2}(r(t)) \\
e_{1}(r(t)) & e_{2}(r(t)) & -b_{3}(r(t))
\end{array}\right) .
$$

Next, we will give several useful definitions and lemmas.

Definition 2.1 ([21]). The SDE (2.1) is said to be stochastically permanent if for any $\varepsilon \in(0,1)$, there exist positive constants $\delta=\delta(\varepsilon)$ and $\chi=\chi(\varepsilon)$ such that

$$
\liminf _{t \rightarrow \infty} P\{|\chi(t)| \leqslant \chi\} \geqslant 1-\varepsilon, \quad \text { and } \liminf _{t \rightarrow \infty} P\{|\chi(t)| \geqslant \delta\} \geqslant 1-\varepsilon,
$$

where $x(t)$ is the solution of (2.1) with any initial value $x\left(\tau_{0}^{+}\right) \in R_{+}^{3}$.

Definition 2.2 ([26]). The $\operatorname{SDE}(2.1)$ is said to be extinct in mean if for any initial value $x\left(\tau_{0}^{+}\right) \in R_{+}^{3}$, solution $x(t)$ of system (2.1) has the property that

$$
\limsup _{t \rightarrow \infty} E|x(t)|=0
$$

Definition 2.3 (Generalized Itô formula [19]). Let $x(t)$ be an $n$-dimensional Itô process on $t \geqslant 0$ with the stochastic differential

$$
d x(t)=f(t) d t+g(t) d B(t),
$$

where $f \in \mathcal{L}^{1}\left(R^{+}, R^{n}\right)$ and $g \in \mathcal{L}^{2}\left(R_{+}, R^{n \times m}\right)$. Let $V \in C^{2,1}\left(R^{n} \times S \times R_{+}, R\right)$, then $V(x(t), r(t), t)$ is a 
real-valued Itô process with its stochastic differential given by

$$
d V(x(t), r(t), t)=\mathcal{L} V(x(t), r(t), t) d t+V_{x}(x(t), r(t), t) g(t) d B(t) \quad \text { a.s., }
$$

where

$$
\begin{aligned}
\mathcal{L} V(x(t), r(t), t)= & V_{t}(x(t), r(t), t)+V_{x}(x(t), r(t), t) f(t) \\
& +\frac{1}{2} \operatorname{trace}\left[g^{\top}(t) V_{x x}(x(t), r(t), t) g(t)\right] \\
& +\sum_{s} \gamma_{r s} V(x(t), s, t) .
\end{aligned}
$$

In addition

$$
\begin{gathered}
V_{t}(x(t), r(t), t)=\frac{\partial V(x(t), r(t), t)}{\partial t}, \\
V_{x}(x(t), r(t), t)=\left(\frac{\partial V(x(t), r(t), t)}{\partial x_{1}}, \cdots, \frac{\partial V(x(t), r(t), t)}{\partial x_{n}}\right),
\end{gathered}
$$

and

$$
V_{x x}(x(t), r(t), t)=\left(\frac{\partial^{2} V(x(t), r(t), t)}{\partial x_{i} \partial x_{j}}\right)_{n \times n} .
$$

Lemma 2.4 ([19, Chebyshev's inequality]). If $c>0, p>0, x \in L^{p}$, then

$$
\mathrm{P}\{\omega:|x(\omega)| \geqslant c\} \leqslant c^{-p} E|x|^{p} .
$$

Lemma 2.5 ([6, Fubini's Theorem $])$. Let $v_{i}(i=1,2)$ be capacities on $A_{i}$ algebras of $\Omega_{i}(i=1,2)$. Let $\Omega=\Omega_{1} \times \Omega_{2}$ be endowed with the product algebra $\mathrm{A}=\mathrm{A}_{1} \otimes A_{2}$. Let $\mathrm{f}: \Omega_{1} \times \Omega_{2} \mapsto \mathrm{R}$ be a slice-comonotonic bounded A-measurable mapping, then

1. $\mathrm{f}\left(\cdot, \omega_{2}\right)$ is $A_{1}$-measurable and $\omega_{2} \in \Omega_{2} \mapsto \int_{\Omega_{1}} f\left(\cdot, \omega_{2}\right) \mathrm{d} \nu_{1}$ is bounded and $A_{2}$-measurable. $f\left(\omega_{1}, \cdot\right)$ is $A_{2}$-measurable and $\omega_{1} \in \Omega_{1} \mapsto \int_{\Omega_{2}} f\left(\omega_{1}, \cdot\right) \mathrm{d} \nu_{2}$ is bounded and $A_{1}$-measurable.

2. The iterated integrals $\iint \mathrm{f} d v_{1} \mathrm{~d} v_{2}, \iint \mathrm{fd} v_{1} \mathrm{~d} v_{2}$ exist and are equal

$$
\int_{\Omega_{2}}\left(\int_{\Omega_{1}} f\left(\omega_{1}, \omega_{2}\right) \mathrm{d} v_{1}\right) \mathrm{d} v_{2}=\int_{\Omega_{1}}\left(\int_{\Omega_{2}} f\left(\omega_{1}, \omega_{2}\right) \mathrm{d} v_{2}\right) \mathrm{d} v_{1}
$$

3. A capacity $v$ on $\left(\Omega_{1} \times \Omega_{2}\right.$, A) satisfies: for any slice-comonotonic bounded A-measurable mapping $f: \Omega_{1} \times \Omega_{2} \rightarrow R$, and

$$
\int \mathrm{fd} v=\iint \mathrm{fd} v_{1} \mathrm{~d} v_{2}=\iint \mathrm{fd} v_{2} \mathrm{~d} v_{1}
$$

if and only if $v$ satisfies $v(A)=\iint A^{*} \mathrm{~d} v_{1} \mathrm{~d} v_{2}$ for any slice-comonotonic $A^{*}$ belonging to $A$. Such a capacity is called a Fubini independent product of $v_{1}$ and $v_{2}$.

Lemma 2.6 ([19, Borel-Cantelli's lemma]).

(1) If $\left\{A_{k}\right\} \subset \mathcal{F}$ and $\sum_{k=1}^{\infty} P\left(A_{k}\right)<\infty$, then

$$
P\left(\limsup _{k \rightarrow \infty} A_{k}\right)=0 .
$$

That is, there exist a set $\Omega_{1} \in \mathcal{F}$ with $\mathrm{P}\left(\Omega_{1}\right)=1$ and an integer-valued random variable $\mathrm{k}_{1}$ such that for every $\omega \in \Omega_{1}$ we have $\omega \notin A_{k}$ whenever $k \geqslant k_{1}(\omega)$.

(2) If the sequence $\left\{A_{k}\right\} \subset \mathcal{F}$ is independent and $\sum_{k=1}^{\infty} P\left(A_{k}\right)=\infty$, then

$$
P\left(\limsup _{k \rightarrow \infty} A_{k}\right)=1 \text {. }
$$

That is, there exists a set $\Omega_{2} \in \mathcal{F}$ with $\mathrm{P}\left(\Omega_{2}\right)=1$ such that for every $\omega \in \Omega_{2}$, there exists a sub-sequence $\left\{A_{k_{i}}\right\}$ such that the $\omega$ belongs to every $A_{k_{i}}$. 


\section{Existence and uniqueness of global positive solution}

As the $x(t)$ determines the species population in the system at time $t$, it should be nonnegative. Moreover, a stochastic differential equation with Markovian switching has a unique global (i.e., no explosion in finite time) solution for any given initial data if the coefficients of the equation satisfy the linear growth condition and Lipschitz condition. The coefficients of SDE (2.1) do not satisfy the linear growth condition, though they are locally Lipschitz continuous. Thus, the solution of SDE (2.1) may explode at a finite time. A necessary condition may establish to ensure the solution of SDE (2.1) is not only positive but will also not explode to infinity at any finite time.

Theorem 3.1. Assume that there exist positive numbers $\mathrm{c}_{1}(\mathrm{I}), \mathrm{c}_{2}(\mathrm{I}), \mathrm{c}_{3}(\mathrm{I})$ for each $\mathrm{I} \in \tilde{\mathrm{S}}$ such that

$$
-\lambda:=\max \left\{\lambda_{\max }^{+}\left(\overline{\mathrm{C}}(\mathrm{I}) \mathrm{A}(\mathrm{I})+\mathrm{A}^{\mathrm{T}}(\mathrm{I}) \overline{\mathrm{C}}(\mathrm{I})\right)\right\} \leqslant 0,
$$

where $\overline{\mathrm{C}}(\mathrm{I})=\operatorname{diag}\left(\mathrm{c}_{1}(\mathrm{I}), \mathrm{c}_{2}(\mathrm{I}), \mathrm{c}_{3}(\mathrm{I})\right)$. Then, for any given initial value $\mathrm{x}\left(\tau_{0}\right) \in \mathrm{R}_{+}^{3}$, there is a unique solution $x(t)$ of system (2.1) defined on $t \in R_{+}$, and remains in $R_{+}^{3}$ with probability one, namely $x(t) \in R_{+}^{3}$ for all $t \in R_{+}$almost surely.

The proof is a modification of the proof for the autonomous case. For the completeness of the paper, we will provide the proof for the cases in Appendix A.

Remark 3.2. Let

$$
\begin{aligned}
J & =\bar{C}(I) A(I)+A^{\top}(I) \bar{C}(I) \\
& =\left(\begin{array}{ccc}
-2 b_{1} c_{1}(I) & -g_{1} c_{1}(I)-g_{2} c_{2}(I) & -d_{1} c_{1}(I)+e_{1} c_{3}(I) \\
-g_{1} c_{1}(I)-g_{2} c_{2}(I) & -2 b_{2} c_{2}(I) & -d_{2} c_{2}(I)+e_{2} c_{3}(I) \\
-d_{1} c_{1}(I)+e_{1} c_{3}(I) & -d_{2} c_{2}(I)+e_{2} c_{3}(I) & -2 b_{3} c_{3}(I)
\end{array}\right) .
\end{aligned}
$$

Its characteristic equation is

$$
\Delta(\lambda)=\lambda^{3}+A_{1} \lambda^{2}+A_{2} \lambda+A_{3},
$$

where the coefficients $A_{1}, A_{2}$ and $A_{3}$ expressed in terms of the matrix are $A_{1}=-\operatorname{tr}(J), A_{3}=-\operatorname{det}(J)$ and $A_{2}=M$, with $M$ indicating the sum of the principal minors of order two of J. Since J is a real symmetric matrix, the characteristic (3.2) has three purely real roots. By Descartes' rule, J has three negative roots is equivalent to

$$
A_{1}>0, \quad A_{1} A_{2}-A_{3}>0, \quad \text { and } \quad A_{3}>0 .
$$

\section{Stochastic permanence to extinction}

Theorem 3.1 states that the solution of (2.1) will remain in the positive cone $R_{+}$. This positivity property of the solution allows the construction of various types of Lyapunov functions to study the dynamic properties of the solution in $R_{+}$in more details. Next, we have the following result, which guarantees the ultimate up boundedness of the solution.

Theorem 4.1. Assume that (3.1) holds. Then there exists a positive number $\mathrm{H}_{1}>0$ such that

$$
\limsup _{t \rightarrow \infty} E|x(t)| \leqslant H_{1}
$$

for any solution $x(t)$ of the system (2.1) with the initial value $x\left(\tau_{0}\right) \in R_{+}^{3}$.

Proof. By Theorem 3.1, the unique solution $x(t)$ of system (2.1) will remain in $R_{+}^{3}$ for all $t \in R_{+}$with probability one. Define a function $V: R_{+}^{3} \times \tilde{S} \rightarrow R_{+}$by

$$
\mathrm{V}(\mathrm{x}, \mathrm{I})=e^{\mathrm{t}}\left[\mathrm{c}_{1}(\mathrm{I}) \mathrm{x}_{1}+\mathrm{c}_{2}(\mathrm{I}) \mathrm{x}_{2}+\mathrm{c}_{3}(\mathrm{I}) \mathrm{y}\right]
$$


By generalized Itô formula, we have

$$
\mathrm{dV}(x, \mathrm{I})=\mathcal{L} \mathrm{V}(x, \mathrm{I}) \mathrm{dt},
$$

where $\mathcal{L} V$ is a mapping from $\mathrm{R}_{+}^{3} \times \tilde{\mathrm{S}} \rightarrow \mathrm{R}$ by

$$
\mathcal{L} V(x, I)=e^{t}\left[C(I) x+x^{\top} \bar{C}(I) a(I)+x^{\top} \bar{C}(I) A(I) x\right]+\sum_{J=1}^{4} \pi_{I J} V(x, J),
$$

where $\mathrm{C}(\mathrm{I})=\left(\mathrm{c}_{1}(\mathrm{I}), \mathrm{c}_{2}(\mathrm{I}), \mathrm{c}_{3}(\mathrm{I})\right)$. By condition (3.1), there is

$$
x^{\top} \bar{C}(I) A(I) x=\frac{x^{\top}\left(\bar{C}(I) A(I)+A^{\top}(I) \bar{C}(I)\right) x}{2} \leqslant-\frac{\lambda}{2}|x|^{2} .
$$

Substituting (4.3) to (4.2), therefore

$$
\begin{aligned}
\mathrm{dV}(x, \mathrm{I}) & =\mathcal{L} V(x, \mathrm{I}) \mathrm{dt} \\
& \leqslant e^{t}\left([|\mathrm{C}(\mathrm{I})|+|\overline{\mathrm{C}}(\mathrm{I}) \mathrm{a}(\mathrm{I})|+4 \pi \mu]|x|-\frac{\lambda}{2}|x|^{2}\right) d t \\
& \leqslant \mathrm{~K}_{1} e^{\mathrm{t}} \mathrm{dt},
\end{aligned}
$$

where $\pi=\max \left\{\pi_{\mathrm{IJ}}, \mathrm{I}, \mathrm{J} \in \widetilde{\mathrm{S}}\right\}, \mu=\max \left\{\frac{\mathrm{c}_{\mathrm{m}}(\mathrm{J})}{\mathrm{c}_{\mathrm{n}}(\mathrm{I})}, 1 \leqslant \mathrm{~m}, \mathrm{n} \leqslant 3, \mathrm{I}, \mathrm{J} \in \widetilde{\mathrm{S}}\right\}, \mathrm{C}(\mathrm{I})=\left(\mathrm{c}_{1}(\mathrm{I}), \mathrm{c}_{2}(\mathrm{I}), \mathrm{c}_{3}(\mathrm{I})\right)$ and $\mathrm{K}_{1}=\max _{\mathrm{I} \in \tilde{\mathrm{S}}} \frac{(|\mathrm{C}(\mathrm{I})|+|\overline{\mathrm{C}}(\mathrm{I}) \mathrm{a}(\mathrm{I})|+\mathrm{p})^{2}}{2 \lambda}>0$.

For any $t \in\left(\tau_{k}, \tau_{k+1}\right]$, integrating both sides of the inequality (4.4) from $\tau_{k}^{+}$to $t$, and then taking expectations yields

$$
\begin{aligned}
\operatorname{EV}(x(t), r(t)) & \leqslant \operatorname{EV}\left(x\left(\tau_{k}^{+}\right), r\left(\tau_{k}^{+}\right)\right)+K_{1}\left(e^{t}-e^{\tau_{k}}\right) \\
& =\operatorname{EV}\left(x\left(\tau_{k}\right), r\left(\tau_{k}\right)\right)+K_{1}\left(e^{t}-e^{\tau_{k}}\right) \\
& \leqslant \operatorname{EV}\left(x\left(\tau_{k-1}^{+}\right), r\left(\tau_{k-1}^{+}\right)\right)+K_{1}\left(e^{\tau_{k}}-e^{\tau_{k-1}}\right)+K_{1}\left(e^{t}-e^{\tau_{k}}\right) \\
& =\operatorname{EV}\left(x\left(\tau_{k-1}\right), r\left(\tau_{k-1}\right)\right)+K_{1}\left(e^{t}-e^{\tau_{k-1}}\right) \\
& \leqslant \operatorname{EV}\left(x\left(\tau_{0}\right), r\left(\tau_{0}\right)\right)+K_{1}\left(e^{t}-e^{\tau_{0}}\right) .
\end{aligned}
$$

Note that

$$
|x(t)| \leqslant \frac{V(x(t), r(t))}{\hat{c} e^{t}} .
$$

Therefore, from (4.5) and (4.6) we obtain

$$
\limsup _{t \rightarrow \infty} E|x(t)| \leqslant K_{1} / \hat{c} \triangleq H_{1},
$$

which means (4.1) holds. The proof of Theorem 4.1 is complete.

Theorem 4.1 illustrates the property of stochastic ultimate boundedness for the solution of system (2.1). Meanwhile, species $x$ needs to be permanent in a realistic setting in the future. Thus, it is necessary for us to research the following conclusion.

Theorem 4.2. Assume that the condition (3.1) holds. In addition, if there exist positive constants $\alpha, \theta$ and $\mathrm{q}(\mathrm{I})$ $(\mathrm{I} \in \tilde{\mathrm{S}})$ such that

$$
\mathrm{q}(\mathrm{I}) \theta \hat{\mathrm{a}}(\mathrm{I})-\sum_{J=1}^{4} \pi_{\mathrm{IJ}} \mathrm{q}(\mathrm{J})-\alpha \mathrm{q}(\mathrm{I})<0,
$$


then the solution $x(t)$ of the system (2.1) with any initial value $x\left(\tau_{0}\right) \in R_{+}^{3}$ has the property that

$$
\limsup _{t \rightarrow \infty} E\left[\frac{1}{|x(t)|^{\theta}}\right] \leqslant H_{2}
$$

here $\mathrm{H}_{2}$ is a positive constant.

Proof. By Theorem 3.1 the unique solution $x(t)$ of system (2.1) will remain in $R_{+}^{3}$ for all $t \in R_{+}$with probability one. Define $V: R_{+}^{3} \rightarrow R_{+}$by

$$
V(x)=x_{1}+x_{2}+y \text { on } t \in R_{+} .
$$

Then

Define also

$$
d V(x)=x^{\top}[a(r(t))+A(r(t)) x] d t
$$

$$
\mathrm{U}(\mathrm{x})=\frac{1}{\mathrm{~V}(\mathrm{x})} \text { on } \mathrm{t} \in \mathrm{R}_{+} .
$$

By generalized Itô formula, we have

$$
\begin{aligned}
\operatorname{du}(x) & =\mathcal{L} U(x) d t \\
& =-U^{2}(x) d V+U^{3}(x)(d V)^{2} \\
& =-U^{2}(x) x^{\top}[a(r(t))+A(r(t)) x] d t .
\end{aligned}
$$

Define a function $\bar{V}: R_{+}^{3} \times \tilde{S} \rightarrow R_{+}$by

$$
\overline{\mathrm{V}}=\mathrm{q}(\mathrm{I})(1+\mathrm{U})^{\Theta},
$$

where for each $I \in \tilde{S}, q(I)>0$. Applying the generalized Itô formula, then

$$
\begin{aligned}
\mathrm{d} \bar{V}= & \mathcal{L} \bar{V} d t \\
= & \left\{\mathrm{q}(\mathrm{I}) \theta(1+\mathrm{U})^{\theta-1}\left[-\mathrm{U}^{2} \chi^{\top}(\mathrm{a}(\mathrm{I})+\mathrm{A}(\mathrm{I}) \mathrm{x})\right]\right. \\
& \left.+\sum_{J=1}^{4} \pi_{\mathrm{IJ}} \mathrm{q}(\mathrm{J})(1+\mathrm{U})^{\theta}\right\} \mathrm{dt} \\
= & \left\{\mathrm{q}(\mathrm{I}) \theta(1+\mathrm{U})^{\theta-2}\left[-(1+\mathrm{U}) \mathrm{U}^{2} \chi^{\top}(\mathrm{a}(\mathrm{I})+\mathrm{A}(\mathrm{I}) \mathrm{x})\right]\right. \\
& \left.+\sum_{J=1}^{4} \pi_{\mathrm{IJ}} \mathrm{q}(\mathrm{J})(1+\mathrm{U})^{\theta}\right\} \mathrm{dt} .
\end{aligned}
$$

We have

$$
\begin{aligned}
& -(1+U) U^{2} x^{\top}(a(I)+A(I) x)=-U^{2} x^{\top} a(I)-U^{3} x^{\top} a(I)-U^{2} x^{\top} A(I) x-U^{3} x^{\top} A(I) x \\
& =-\frac{x^{\top} A(I) x}{V^{2}}+\left[-\frac{x^{\top} a(I)}{V}-\frac{x^{\top} A(I) x}{V^{2}}\right] U-\left[\frac{x^{\top} a(I)}{V}\right] U^{2} \text {. }
\end{aligned}
$$

It is easy to see that for all $x \in R_{+}^{3}$,

$$
-\frac{x^{\top} A(I) x}{V^{2}} \leqslant K_{2}, \quad \text { and } \quad-\frac{x^{\top} a(I)}{V}-\frac{x^{\top} A(I) x}{V^{2}} \leqslant K_{2},
$$

where $K_{2}$ is a positive constant, while

$$
\frac{x^{\top} a(I)}{V} \geqslant \hat{a}(I)
$$


Hence

$$
-(1+U) U^{2} x^{\top}(a(I)+A(I) x) \leqslant K_{2}+K_{2} U-\hat{a}(I) U^{2} .
$$

Substituting above inequality to (4.9) yields

$$
\begin{aligned}
\mathcal{L} \bar{V} \leqslant & q(I) \theta(1+\mathrm{U})^{\theta-2}\left[-\hat{a}(\mathrm{I}) \mathrm{U}^{2}+\mathrm{K}_{2}(1+\mathrm{U})\right]+\sum_{J=1}^{4} \pi_{\mathrm{IJ}} \mathrm{q}(\mathrm{J})(1+\mathrm{U})^{\theta} \\
= & (1+\mathrm{U})^{\theta-2}\left\{-\left[\mathrm{q}(\mathrm{I}) \theta \hat{\mathrm{a}}(\mathrm{I})-\sum_{J=1}^{4} \pi_{\mathrm{IJ}} \mathrm{q}(\mathrm{J})\right] \mathrm{U}^{2}+\left[\mathrm{q}(\mathrm{I}) \theta \mathrm{K}_{2}\right.\right. \\
& \left.\left.+2 \sum_{J=1}^{4} \pi_{\mathrm{IJ}} \mathrm{q}(\mathrm{J})\right] \mathrm{U}+\mathrm{q}(\mathrm{I}) \theta \mathrm{K}_{2}+\sum_{J=1}^{4} \pi_{\mathrm{IJ}} \mathrm{q}(\mathrm{J})\right\} .
\end{aligned}
$$

Let $\alpha>0$, and hence

$$
\begin{aligned}
\mathcal{L} e^{\alpha t} \bar{V}= & \alpha e^{\alpha t} q(I)(1+\mathrm{U})^{\theta}+e^{\alpha t} \mathcal{L} \bar{V} \\
\leqslant & e^{\alpha t}(1+\mathrm{U})^{\theta-2}\left\{\alpha q(I)(1+\mathrm{U})^{2}-\left[\mathrm{q}(\mathrm{I}) \theta \hat{a}(\mathrm{I})-\sum_{J=1}^{4} \pi_{I J} \mathrm{q}(\mathrm{J})\right] \mathrm{U}^{2}\right. \\
& \left.+\left[\mathrm{q}(\mathrm{I}) \theta \mathrm{K}_{2}+2 \sum_{J=1}^{4} \pi_{\mathrm{IJ}} \mathrm{q}(\mathrm{J})\right] \mathrm{U}+\mathrm{q}(\mathrm{I}) \theta \mathrm{K}_{2}+\sum_{J=1}^{4} \pi_{\mathrm{IJ}} \mathrm{q}(\mathrm{J})\right\} \\
= & e^{\alpha \mathrm{t}}(1+\mathrm{U})^{\theta-2}\left\{-\mathrm{U}^{2}\left[\mathrm{q}(\mathrm{I}) \theta \hat{\mathrm{a}}(\mathrm{I})-\sum_{J=1}^{4} \pi_{\mathrm{IJ}} \mathrm{q}(\mathrm{J})-\alpha \mathrm{q}(\mathrm{I})\right]\right. \\
& +\left[\mathrm{q}(\mathrm{I}) \theta \mathrm{K}_{2}+2 \sum_{J=1}^{4} \pi_{\mathrm{IJ}} \mathrm{q}(\mathrm{J})+2 \alpha \mathrm{q}(\mathrm{I})\right] \mathrm{U}+\mathrm{q}(\mathrm{I}) \theta \mathrm{K}_{2} \\
& \left.+\sum_{J=1}^{4} \pi_{\mathrm{IJ}} \mathrm{q}(\mathrm{J})+\alpha \mathrm{q}(\mathrm{I})\right\} \\
\leqslant & 3^{-\theta} \hat{\mathrm{q}}(\mathrm{I}) \mathrm{H} e^{\alpha t},
\end{aligned}
$$

where

$$
\begin{aligned}
H= & \frac{1}{\hat{q}(I)} 3^{\theta} \max \left\{\operatorname { s u p } \left\{( 1 + \mathrm { U } ) ^ { \theta - 2 } \left\{-\mathrm{U}^{2}\left[\mathrm{q}(\mathrm{I}) \theta \hat{\mathrm{a}}(\mathrm{I})-\sum_{J=1}^{4} \pi_{\mathrm{IJ}} \mathrm{q}(\mathrm{J})-\alpha \mathrm{q}(\mathrm{I})\right]\right.\right.\right. \\
& +\left[\mathrm{q}(\mathrm{I}) \theta \mathrm{K}_{2}+2 \sum_{\mathrm{J}=1}^{4} \pi_{\mathrm{IJ}} \mathrm{q}(\mathrm{J})+2 \alpha \mathrm{q}(\mathrm{I})\right] \mathrm{U}+\mathrm{q}(\mathrm{I}) \theta \mathrm{K}_{2} \\
& \left.\left.\left.+\sum_{J=1}^{4} \pi_{\mathrm{IJ}} \mathrm{q}(\mathrm{J})+\alpha \mathrm{q}(\mathrm{I})\right\}\right\}, 1\right\},
\end{aligned}
$$

in which we put 1 in order to make $\mathrm{H}$ positive. The inequality (4.10) implies

$$
\limsup _{t \rightarrow \infty} E\left[U^{\theta}(x(t))\right] \leqslant \limsup _{t \rightarrow \infty} E\left[(1+U(x(t)))^{\theta}\right] \leqslant 3^{-\theta} H .
$$

For $x(t) \in R_{+}^{3}$, note that

$$
\left(x_{1}+x_{2}+y\right)^{\theta} \leqslant\left(3 \max \left\{x_{1}, x_{2}, y\right\}\right)^{\theta}=3^{\theta}\left(\max \left\{x_{1}^{2}, x_{2}^{2}, y^{2}\right\}\right)^{\frac{\theta}{2}} \leqslant 3^{\theta}|x(t)|^{\theta} .
$$


Consequently,

$$
\limsup _{t \rightarrow \infty} E\left[\frac{1}{|x(t)|^{\theta}}\right] \leqslant H_{2} .
$$

This completes the proof of Theorem 4.2.

Theorems 4.1 and 4.2 show that the solution of system (2.1) is stochastically bounded. Under the circumstances, we have the following result about permanence by applying Lemma 2.4.

Theorem 4.3. Assume that all conditions of Theorems 4.1 and 4.2 hold, then any positive solution $x(t)$ of system (2.1) with the initial value $x\left(\tau_{0}\right) \in R_{+}^{3}$ is stochastically permanent.

Proof. By Theorem 4.1, we derive that

$$
\limsup _{t \rightarrow \infty} E|x(t)| \leqslant H_{1} .
$$

For any $\varepsilon>0$, assign $\delta_{1}=\frac{\mathrm{H}_{1}}{\varepsilon}$, by Lemma 2.4 one has

$$
\mathrm{P}\left\{|x(\mathrm{t})|>\delta_{1}\right\} \leqslant \frac{\mathrm{E}|x(\mathrm{t})|}{\delta_{1}} .
$$

Hence

$$
\limsup _{t \rightarrow \infty} P\left\{|x(t)|>\delta_{1}\right\} \leqslant \varepsilon
$$

Then, we have

$$
\liminf _{t \rightarrow \infty} P\left\{|x(t)|>\delta_{1}\right\} \leqslant \limsup _{t \rightarrow \infty} P\left\{|x(t)|>\delta_{1}\right\} \leqslant \varepsilon .
$$

This implies

$$
\liminf _{t \rightarrow \infty} P\left\{|x(t)| \leqslant \delta_{1}\right\} \geqslant 1-\varepsilon
$$

By Theorem 4.2, we have

$$
\limsup _{t \rightarrow \infty} E\left[\frac{1}{\left|x(t)^{\theta}\right|}\right] \leqslant H_{2}
$$

Then, for any $\varepsilon>0$, let $\delta_{2}=\varepsilon / \mathrm{H}_{2}^{\frac{1}{\theta}}$, by Lemma 2.4 we obtain

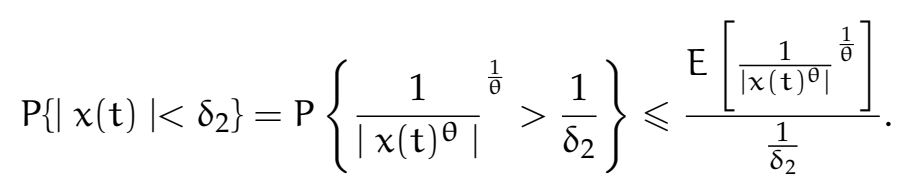

Thus

$$
\limsup _{t \rightarrow \infty} P\left\{|x(t)|<\delta_{2}\right\} \leqslant \varepsilon
$$

That is

$$
\liminf _{t \rightarrow \infty} P\left\{|x(t)| \geqslant \delta_{2}\right\} \geqslant 1-\varepsilon,
$$

which together with (4.12) yields that system (2.1) is stochastic permanent. The proof of Theorem 4.3 is complete.

Theorem 4.3 demonstrates the property of stochastic permanence. Species may also become extinct under some special circumstance such as resource shortages or major environmental changes, which play a vital role in the study of ecology systems. Hence, we arrive at the result that all species of system (2.1) will be extinct in mean. 
Theorem 4.4. Assume that condition (3.1) holds. For any initial value $x\left(\tau_{0}\right) \in R_{+}^{3}$, the solution $x(t)$ of the system (2.1) has the property that

$$
\limsup _{t \rightarrow \infty} \frac{\ln |x(t)|}{t} \leqslant \pi_{I I}(1-\mu)+\limsup _{t \rightarrow \infty} \frac{1}{t} \int_{\tau_{0}}^{t} \check{a}(r(s)) d s \quad \text { a.s.. }
$$

Particularly, if

$$
\pi_{\text {II }}(1-\mu)+\limsup _{t \rightarrow \infty} \frac{1}{t} \int_{\tau_{0}}^{t} \check{a}(r(s)) d s<0,
$$

then

$$
\lim _{t \rightarrow \infty}|x(t)|=0 \quad \text { a.s.. }
$$

Proof. By condition (3.1), the unique solution $x(t)$ of system (2.1) will remain in $R_{+}^{3}$ for all $t \in R_{+}$with probability one. Define a function $V: R_{+}^{3} \times \tilde{S} \rightarrow R_{+}$by

$$
\mathrm{V}(\mathrm{x}, \mathrm{I})=\mathrm{c}_{1}(\mathrm{I}) \mathrm{x}_{1}+\mathrm{c}_{2}(\mathrm{I}) \mathrm{x}_{2}+\mathrm{c}_{3}(\mathrm{I}) \mathrm{y} \text {. }
$$

By generalized Itô formula, we derive from (4.15) that

$$
\begin{aligned}
\operatorname{dV}(x, I) & =\mathcal{L} V(x, I) d t \\
& =\left\{x^{\top} \bar{C}(I)[a(I)+A(I) x]+\sum_{J=1}^{4} \pi_{I J} V(x, J)\right\} d t .
\end{aligned}
$$

Then

$$
\begin{aligned}
d \ln V(x, I) & =\frac{1}{V(x, I)} d V(x, I)-\frac{1}{2 V^{2}}(d V(x, I))^{2} \\
& =\frac{1}{V(x, I)}\left\{x^{\top} \bar{C}(I)[a(I)+A(I) x]+\sum_{J=1}^{4} \pi_{I J} V(x, J)\right\} d t .
\end{aligned}
$$

From (4.15) and (3.1) that

$$
\begin{aligned}
\frac{x^{\top} \overline{\mathrm{C}}(\mathrm{I}) \mathrm{A}(\mathrm{I}) x}{\mathrm{~V}(x, \mathrm{I})} & =\frac{x^{\top}\left(\overline{\mathrm{C}}(\mathrm{I}) \mathrm{A}(\mathrm{I})+\mathrm{A}^{\top}(\mathrm{I}) \overline{\mathrm{C}}(\mathrm{I})\right) x}{2 \mathrm{~V}(x, \mathrm{I})} \\
& \leqslant \frac{-\lambda|x|^{2}}{2 \mathrm{~V}(x, \mathrm{I})} \leqslant \frac{-\lambda}{2|\mathrm{C}(\mathrm{I})|}|x| \leqslant 0 .
\end{aligned}
$$

Therefore,

$$
\begin{aligned}
\frac{x^{\top} \overline{\mathrm{C}}(\mathrm{I}) \mathrm{a}(\mathrm{I})}{\mathrm{V}(\mathrm{x}, \mathrm{I})}+\frac{\sum_{\mathrm{J}=1}^{4} \pi_{\mathrm{IJ}} \mathrm{V}(x, \mathrm{~J})}{\mathrm{V}(\mathrm{x}, \mathrm{I})} & \leqslant \check{\mathrm{a}}(\mathrm{I})+\frac{\pi_{\mathrm{II}} \mathrm{V}(x, \mathrm{I})}{\mathrm{V}(x, \mathrm{I})}+\frac{\sum_{\mathrm{J} \neq \mathrm{I}} \pi_{\mathrm{IJ}} \mathrm{V}(x, \mathrm{~J})}{\mathrm{V}(x, \mathrm{I})} \\
& =\check{\mathrm{a}}(\mathrm{I})+\pi_{\mathrm{II}}+\frac{\sum_{\mathrm{J} \neq \mathrm{I}} \pi_{\mathrm{IJ}} \mathrm{V}(x, \mathrm{~J})}{\mathrm{V}(x, \mathrm{I})} \\
& \leqslant \check{\mathrm{a}}(\mathrm{I})+\pi_{\mathrm{II}}-\frac{\mu \pi_{\mathrm{II}} \mathrm{V}(x, \mathrm{I})}{\mathrm{V}(x, \mathrm{I})} \\
& =\check{\mathrm{a}}(\mathrm{I})+\pi_{\mathrm{II}}(1-\mu) .
\end{aligned}
$$

Substituting (4.17) and (4.18) into (4.16) yields

$$
\mathrm{d} \ln \mathrm{V}(\mathrm{x}, \mathrm{I}) \leqslant \check{\mathrm{a}}(\mathrm{I})+\pi_{\mathrm{II}}(1-\mu) .
$$

Integrating both sides of the inequality (4.19) from $\tau_{0}$ to $t$, then

$$
\ln V(x(t), r(t)) \leqslant \ln V\left(x\left(\tau_{0}\right), r\left(\tau_{0}\right)\right)+\int_{\tau_{0}}^{t}\left[\check{a}(r(s))+\pi_{I I}(1-\mu)\right] d s .
$$


It finally follows from (4.20) by dividing $t$ on the both sides, and taking superior limit, we have

$$
\limsup _{t \rightarrow \infty} \frac{\ln V(x(t))}{t} \leqslant \pi_{I I}(1-\mu)+\limsup _{t \rightarrow \infty} \frac{1}{t} \int_{\tau_{0}}^{t} \check{a}(r(s)) d s, \quad \text { a.s., }
$$

which implies the required assertions (4.13) and (4.14). This completes the proof of Theorem 4.4.

\section{Path-wise estimation}

Theorem 5.1. Assume that the condition (3.1) holds. Then for any initial value $x\left(\tau_{0}\right) \in R_{+}^{3}$, any solution $x(t)$ of system (2.1) has the property that

$$
\limsup _{t \rightarrow \infty} \frac{\ln |x(t)|}{\ln t} \leqslant 1 \quad \text { a.s.. }
$$

Proof. Let $\mathrm{V}: \mathrm{R}_{+}^{3} \times \tilde{S} \rightarrow \mathrm{R}_{+}$be defined as (4.15), by the generalized Itô formula, we can show that

$$
\mathcal{L} \mathrm{V}=\left(x^{\top} \overline{\mathrm{C}}(\mathrm{I})[\mathrm{a}(\mathrm{I})+\mathrm{A}(\mathrm{I}) \mathrm{x}]+\sum_{\mathrm{J}=1}^{4} \pi_{\mathrm{IJ}} \mathrm{V}(\mathrm{x}, \mathrm{J})\right),
$$

which from (3.1) we know $x^{\top} \bar{C}(I) A(I) x \leqslant-\frac{\lambda}{2}|x|^{2}<0$.

Substituting above inequality, $\sum_{J=1}^{4} \pi_{I J} V(x, J) \leqslant 4 \pi \mu V(x, I)$ and $x^{\top} \bar{C}(I) a(I) \leqslant|\bar{C}(I) a(I) \| x|$ into (5.2), we obtain

$$
\mathcal{L} V \leqslant|\bar{C}(I) a(I)||x|+4 \pi \mu V(x, I) .
$$

Then

$$
\begin{aligned}
E\left(\sup _{t \leqslant r \leqslant t+1} V(x(r), r(r)) \leqslant\right. & E V(x(t), r(t))+\max _{I \in \tilde{S}}|\bar{C}(I) a(I)| \int_{t}^{t+1} E(|x(s)|) d s \\
& +4 \pi \mu \int_{t}^{t+1} E V(x(s)) d s .
\end{aligned}
$$

From (4.1) of Theorem 4.1, we know that,

$$
\limsup _{t \rightarrow \infty} E\left(\sup _{t \leqslant r \leqslant(t+1)} V(x(t), r(t))\right) \leqslant \sqrt{3} \breve{c}(I) \limsup _{t \rightarrow \infty} E(|x(t)|) \leqslant \sqrt{3} \breve{c}(I) H_{1} .
$$

Therefore

$$
\begin{aligned}
\limsup _{\mathrm{t} \rightarrow \infty} E\left(\sup _{\mathrm{t} \leqslant r \leqslant t+1} V(x(r), r(r))\right) \leqslant & \sqrt{3} \breve{c}(I) H_{1}+\max _{\mathrm{I} \in \tilde{\mathrm{S}}}|\overline{\mathrm{C}}(\mathrm{I}) \mathrm{a}(\mathrm{I})| \mathrm{H}_{1} \\
& +4 \sqrt{3} \pi \mu \breve{c}(\mathrm{I}) \mathrm{H}_{1} \\
= & {\left[(4 \pi \mu+1) \sqrt{3} \breve{\mathrm{c}}+\max _{\mathrm{I} \in \tilde{\mathrm{S}}}|\overline{\mathrm{C}}(\mathrm{I}) \mathrm{a}(\mathrm{I})|\right] \mathrm{H}_{1} . }
\end{aligned}
$$

Recalling the following inequality

$$
|x(t)| \leqslant x_{1}+x_{2}+y \leqslant \frac{1}{\hat{c}(I)} V(x, I), \quad \forall x(t) \in R_{+}^{3},
$$

we obtain

$$
\limsup _{t \rightarrow \infty} E\left(\sup _{t \leqslant r \leqslant t+1}|x(r)|\right) \leqslant \frac{1}{\hat{c}(I)}\left[(4 \pi \mu+1) \sqrt{3} \breve{c}+\max _{I \in \tilde{S}}|\bar{C}(I) a(I)|\right] H_{1} .
$$

We can observe from (5.3) that there is a positive constant $\overline{\mathrm{H}}$ such that

$$
E\left(\sup _{k \leqslant t \leqslant k+1}|x(t)|\right) \leqslant \bar{H}, \quad k=1,2, \cdots .
$$


Let $\epsilon>0$ be arbitrary. Then, by Lemma 2.4, we have

$$
P\left\{\sup _{k \leqslant t \leqslant k+1}|x(t)|>k^{1+\epsilon}\right\} \leqslant \frac{\bar{H}}{k^{1+\epsilon}}, \quad k=1,2, \cdots .
$$

Applying Lemma 2.6, we obtain that for almost all $\omega \in \Omega$

$$
\sup _{k \leqslant t \leqslant k+1}|x(k)| \leqslant k^{1+\epsilon}
$$

holds for all but finitely many $k$. Hence, there exists a $k_{0}(\omega)$ for almost all $\omega \in \Omega$, such that (5.3) holds whenever $k \geqslant k_{0}$. Consequently, for almost all $\omega \in \Omega$, if $k \geqslant k_{0}$ and $k \leqslant t \leqslant k+1$,

$$
\frac{\ln (|x(t)|)}{\ln t} \leqslant \frac{(1+\epsilon) \ln k}{\ln k}=1+\epsilon .
$$

Therefore

$$
\limsup _{t \rightarrow \infty} \frac{\ln (|x(t)|)}{\ln t} \leqslant 1+\epsilon . \quad \text { a.s.. }
$$

Letting $\epsilon \rightarrow 0$, we obtain the assertion (5.1). This completes the proof of Theorem 5.1.

Theorem 5.2. If conditions (3.1) and (4.7) hold, then any positive solution $x(t)$ of system (2.1) with any initial value $x\left(\tau_{0}\right) \in R_{+}^{3}$ has the property that

$$
\liminf _{t \rightarrow \infty} \frac{\ln (|x(t)|)}{\ln t} \geqslant-\frac{1}{\theta} \quad \text { a.s.. }
$$

Proof. Let $\mathrm{U}: \mathrm{R}_{+}^{3} \rightarrow \mathrm{R}_{+}$be the same as defined by (4.8), for convenience, we write $\mathrm{U}(\mathrm{x}(\mathrm{t}))=\mathrm{U}(\mathrm{t})$. Applying the generalized Itô formula, for the fixed constant $\theta>0$, we derive

$$
\begin{aligned}
\mathcal{L} \mathrm{q}(\mathrm{I})(1+\mathrm{U}(\mathrm{t}))^{\theta} \leqslant & \mathrm{q}(\mathrm{I}) \theta(1+\mathrm{U}(\mathrm{t}))^{\theta-2}\left\{-\left[\mathrm{q}(\mathrm{I}) \theta \hat{\mathrm{a}}(\mathrm{I})-\sum_{J=1}^{4} \pi_{\mathrm{IJ}} \mathrm{q}(\mathrm{J})\right] \mathrm{U}^{2}(\mathrm{t})\right. \\
& +\left(\mathrm{q}(\mathrm{I}) \theta \mathrm{K}_{2}+2 \sum_{\mathrm{J}=1}^{4} \pi_{\mathrm{IJ}} \mathrm{q}(\mathrm{J})\right) \mathrm{u}(\mathrm{t})+\mathrm{q}(\mathrm{I}) \theta \mathrm{K}_{2} \\
& \left.+\sum_{J=1}^{4} \pi_{\mathrm{IJ}} \mathrm{q}(\mathrm{J})\right\} .
\end{aligned}
$$

Let

$$
\bar{\alpha}=\max \left\{\left|\mathrm{q}(\mathrm{I}) \theta \mathrm{K}_{2}+2 \sum_{J=1}^{4} \pi_{\mathrm{IJ}} \mathrm{q}(\mathrm{J})\right|,\left|\mathrm{q}(\mathrm{I}) \theta \mathrm{K}_{2}+\sum_{J=1}^{4} \pi_{\mathrm{IJ}} \mathrm{q}(\mathrm{J})\right|\right\} .
$$

Then

$$
(1+\mathrm{U}(\mathrm{t}))^{\theta-2}\left\{-\left[\mathrm{q}(\mathrm{I}) \theta \hat{\mathrm{a}}(\mathrm{I})-\sum_{J=1}^{4} \pi_{\mathrm{IJ}} \mathrm{q}(\mathrm{J})\right] \mathrm{U}^{2}(\mathrm{t})+\bar{\alpha}(1+\mathrm{U}(\mathrm{t}))\right\} .
$$

Under given condition, by (4.11) of Theorem 4.2, there exists a positive constant $M$ such that

$$
E\left(q(I)(1+U(t))^{\theta}\right) \leqslant M \text { on } t \geqslant 0 .
$$

Let $\delta>0$ such that

$$
\left[\sum_{J=1}^{4} \pi_{I J} \mathrm{q}(\mathrm{J})+\mathrm{q}(\mathrm{I}) \theta \hat{\mathrm{a}}(\mathrm{I})+\bar{\alpha}\right] \delta \leqslant \frac{1}{2}
$$


Letting $k=1,2,3 \cdots,(5.5)$ implies that

$$
\begin{aligned}
E\left[\sup _{(k-1) \delta \leqslant t \leqslant k \delta} q(I)(1+U(t))^{\theta}\right] \leqslant & q(I)(1+U((k-1) \delta))^{\theta}+E\left(\sup _{(k-1) \delta \leqslant t \leqslant k \delta} \mid \int_{(k-1) \delta}^{t}(1+U(s))^{\theta-2}\right. \\
& \left.\times\left\{\left(\sum_{J=1}^{4} \pi_{I J} q(J)-q(I) \theta \hat{a}(I)\right) U^{2}(s)+\bar{\alpha}(1+U(s))\right\} d s \mid\right) .
\end{aligned}
$$

We have

$$
\begin{aligned}
& \mathrm{E}\left(\sup _{(\mathrm{k}-1) \delta \leqslant \mathrm{t} \leqslant \mathrm{k} \delta}\left|\int_{(\mathrm{k}-1) \delta}^{\mathrm{t}}(1+\mathrm{U}(\mathrm{s}))^{\theta-2}\left\{\left(\sum_{\mathrm{J}=1}^{4} \pi_{\mathrm{IJ}} \mathrm{q}(\mathrm{J})-\mathrm{q}(\mathrm{I}) \theta \hat{\mathrm{a}}(\mathrm{I})\right) \mathrm{U}^{2}(\mathrm{~s})+\bar{\alpha}(1+\mathrm{U}(\mathrm{s}))\right\} \mathrm{ds}\right|\right) \\
& \leqslant \mathrm{E}\left(\int_{(\mathrm{k}-1) \delta}^{\mathrm{k} \delta}\left|(1+\mathrm{U}(\mathrm{s}))^{\theta-2}\left\{\left(\mathrm{q}(\mathrm{I}) \theta \hat{\mathrm{a}}(\mathrm{r}(\mathrm{s}))+\sum_{\mathrm{J}=1}^{4} \pi_{\mathrm{IJ}} \mathrm{q}(\mathrm{J})\right) \mathrm{U}^{2}(\mathrm{~s})+\bar{\alpha}(1+\mathrm{U}(\mathrm{s}))\right\}\right| \mathrm{d} s\right) \\
& \leqslant \mathrm{E}\left(\int_{(k-1) \delta}^{\mathrm{k} \delta} \sup _{(k-1) \delta \leqslant s \leqslant k \delta}\left[\left(\mathrm{q}(\mathrm{I}) \theta \hat{\mathrm{a}}(\mathrm{r}(\mathrm{s}))+\sum_{\mathrm{J}=1}^{4} \pi_{\mathrm{IJ}} \mathrm{q}(\mathrm{J})+\bar{\alpha}\right] \cdot(1+\mathrm{U}(\mathrm{s}))^{\theta} \mathrm{ds}\right)\right. \\
& \leqslant\left(\sum_{J=1}^{4} \pi_{I J} \mathrm{q}(\mathrm{J})+\mathrm{q}(\mathrm{I}) \theta \hat{\mathrm{a}}(\mathrm{I})+\bar{\alpha}\right) \mathrm{E}\left(\int_{(\mathrm{k}-1) \delta}^{\mathrm{k} \delta} \sup (1+\mathrm{U}(\mathrm{s}))^{\theta} \mathrm{ds}\right) \\
& \leqslant\left(\sum_{J=1}^{4} \pi_{I J} \mathrm{q}(\mathrm{J})+\mathrm{q}(\mathrm{I}) \hat{\mathrm{a}}(\mathrm{I})+\bar{\alpha}\right) \delta \mathrm{E}\left(\sup _{(\mathrm{k}-1) \delta \leqslant \mathrm{t} \leqslant \mathrm{k} \delta}(1+\mathrm{U}(\mathrm{t}))^{\theta}\right) \text {. }
\end{aligned}
$$

Therefore

$$
\begin{gathered}
E\left(\sup _{(k-1) \delta \leqslant t \leqslant k \delta}(1+\mathrm{U}(\mathrm{t}))^{\theta}\right) \leqslant\left[(1+\mathrm{U}((\mathrm{k}-1) \delta))^{\theta}\right]+\frac{1}{2} \mathrm{E}\left(\sup _{(\mathrm{k}-1) \delta \leqslant t \leqslant k \delta}(1+\mathrm{U}(\mathrm{t}))^{\theta}\right), \\
\mathrm{E}\left[\sup _{(\mathrm{k}-1) \delta \leqslant t \leqslant k \delta}(1+\mathrm{U}(\mathrm{t}))^{\theta}\right] \leqslant 2 M .
\end{gathered}
$$

Let $\epsilon>0$ be arbitrary. Then, by Lemma 2.4, we have

$$
P\left\{\omega: \sup _{(k-1) \delta \leqslant t \leqslant k \delta}(1+\mathrm{U}(\mathrm{t}))^{\Theta}>(k \delta)^{1+\epsilon}\right\} \leqslant \frac{2 M}{(k \delta)^{1+\epsilon}}, \quad k=1,2, \cdots .
$$

Applying Lemma 2.6, we obtain that all $\omega \in \Omega$ for which (5.6) holds whenever $k \geqslant k_{0}$. Consequently, for almost all $\omega \in \Omega$, if $k \geqslant k_{0}$ and $(k-1) \delta \leqslant t \leqslant k \delta$,

$$
\frac{\ln (1+\mathrm{U}(\mathrm{t}))^{\theta}}{\ln \mathrm{t}} \leqslant \frac{(1+\epsilon) \ln (\mathrm{k} \delta)}{\ln ((\mathrm{k}-1) \delta)}=1+\epsilon .
$$

Therefore

$$
\limsup _{t \rightarrow \infty} \frac{\ln (1+\mathrm{U}(\mathrm{t}))^{\Theta}}{\ln t} \leqslant 1+\epsilon . \quad \text { a.s.. }
$$

Letting $\epsilon \rightarrow 0$, we obtain

$$
\limsup _{t \rightarrow \infty} \frac{\ln (1+\mathrm{U}(\mathrm{t}))^{\Theta}}{\ln t} \leqslant 1 . \quad \text { a.s.. }
$$

Recalling the definition of $\mathrm{u}(\mathrm{t})$, we yield

$$
\limsup _{t \rightarrow \infty} \frac{\ln \frac{1}{|x(t)|^{\theta}}}{\ln t} \leqslant 1 \text {. a.s., }
$$


which further implies

$$
\liminf _{t \rightarrow \infty} \frac{\ln |x(t)|}{\ln t} \geqslant-\frac{1}{\theta} . \text { a.s.. }
$$

This completes the proof of Theorem 5.2.

Theorem 5.3. Under conditions (3.1) and (4.7), for any initial value $x\left(\tau_{0}\right) \in R_{+}^{3}$, the solution $x(t)$ of system (2.1) obeys

$$
\begin{aligned}
& \limsup _{t \rightarrow \infty} \frac{1}{t} \int_{0}^{t}|x(s)| \mathrm{d} s \leqslant \frac{2|C(I)|}{\lambda}\left[\pi_{\mathrm{II}}(1-\mu)+\limsup _{t \rightarrow \infty} \frac{1}{t} \int_{0}^{t} \check{a}(r(s)) \mathrm{d} s\right] \quad \text { a.s., } \\
& \liminf _{t \rightarrow \infty} \frac{1}{t} \int_{0}^{t}|x(s)| \mathrm{d} s \geqslant \frac{2 \hat{c}(I)}{\hat{\lambda}}\left[\pi_{I I}(1-\mu)+\liminf _{t \rightarrow \infty} \frac{1}{t} \int_{0}^{t} \hat{a}(r(s)) d s\right] \quad \text { a.s., }
\end{aligned}
$$

where $\bar{\mu}=\min \left\{\frac{c_{m}(J)}{c_{n}(I)}: 1 \leqslant m, n \leqslant 3, I, J \in \tilde{S}\right\},-\hat{\lambda}:=\min \left\{\lambda_{\max }^{+}\left(\bar{C}(I) A(I)+A^{\top}(I) \bar{C}(I)\right)\right\}$.

Proof. Define $\mathrm{V}: \mathrm{R}_{+}^{3} \times \tilde{\mathrm{S}} \rightarrow \mathrm{R}_{+}$by the relation (4.15). By the generalized Itô formula, we have

$$
\mathrm{dV}(x, \mathrm{I})=\left\{x^{\mathrm{t}} \overline{\mathrm{C}}(\mathrm{I})[\mathrm{a}(\mathrm{I})+\mathrm{A}(\mathrm{I}) \mathrm{x}(\mathrm{t})]+\sum_{\mathrm{J}=1}^{4} \pi_{\mathrm{IJ}} \mathrm{q}(\mathrm{J}) \mathrm{V}(\mathrm{x}, \mathrm{J})\right\} \mathrm{dt} .
$$

It is easy to observe from the inequalities (5.1) and (5.4) that

$$
\lim _{t \rightarrow \infty} \frac{\ln V(x(t), r(t))}{t}=0 \quad \text { a.s.. }
$$

We derive from (5.9) that

$$
V(x, I)=\frac{1}{V(x, I)}\left\{x^{\top} \bar{C}(I)[a(I)+A(I) x(t)]+\sum_{J=1}^{4} \pi_{I J} q(J) V(x, J)\right\} .
$$

By condition (3.1), we have

$$
\frac{-\hat{\lambda}}{2 \hat{\mathrm{c}}(\mathrm{I})}|x|<\frac{x^{\top} \overline{\mathrm{C}}(\mathrm{I}) \mathrm{A}(\mathrm{I}) x}{\mathrm{~V}(x, \mathrm{I})}=\frac{x^{\top}\left[\mathrm{C}(\mathrm{I}) \mathrm{A}(\mathrm{I})+\mathrm{A}^{\top} \overline{\mathrm{C}}(\mathrm{I})\right] x}{2 \mathrm{~V}(x, \mathrm{I})} \leqslant \frac{-\lambda}{2|\mathrm{C}(\mathrm{I})|}|x|<0,
$$

and

$$
\hat{\mathrm{a}}(\mathrm{I})+\pi_{\mathrm{II}}(1-\bar{\mu}) \leqslant \frac{x^{\top} \overline{\mathrm{C}}(\mathrm{I}) \mathrm{a}(\mathrm{I})}{\mathrm{V}(\mathrm{x}, \mathrm{I})}+\frac{\sum_{\mathrm{J}=1}^{4} \pi_{\mathrm{IJ}} \mathrm{q}(\mathrm{J}) \mathrm{V}(\mathrm{x}, \mathrm{J})}{\mathrm{V}(\mathrm{x}, \mathrm{I})} \leqslant \check{\mathrm{a}}(\mathrm{I})+\pi_{\mathrm{II}}(1-\mu) .
$$

Substituting (5.11) and (5.12) to (5.10) yields

$$
\mathrm{d} \ln \mathrm{V}(\mathrm{x}, \mathrm{I}) \leqslant\left[\breve{\mathrm{a}}(\mathrm{I})+\pi_{\mathrm{II}}(1-\mu)-\frac{\lambda}{2|\mathrm{C}(\mathrm{I})|}|x|\right] \mathrm{dt} .
$$

Thus

$$
\ln \mathrm{V}(\mathrm{x}(\mathrm{t}), \mathrm{r}(\mathrm{t}))+\frac{\lambda}{2|\mathrm{C}(\mathrm{I})|} \int_{0}^{\mathrm{t}}|x(\mathrm{~s})| \mathrm{d} s \leqslant \ln \mathrm{V}\left(\mathrm{x}\left(\tau_{0}\right), \mathrm{r}\left(\tau_{0}\right)\right)+\int_{0}^{\mathrm{t}}\left[\pi_{\mathrm{II}}(1-\mu)+\check{\mathrm{a}}(\mathrm{r}(\mathrm{s}))\right] \mathrm{d} \mathrm{s},
$$

we can therefore divide both sides of (5.12) by $t$ and then let $t \rightarrow \infty$ to obtain

$$
\frac{\lambda}{2|C(I)|} \limsup _{t \rightarrow \infty} \frac{1}{t} \int_{0}^{t}|x(s)| d s \leqslant \pi_{I I}(1-\mu)+\limsup _{t \rightarrow \infty} \frac{1}{t} \int_{0}^{t} \check{a}(r(s)) d s .
$$

This completes the proof of (5.7).

On the other hand, we observe from (5.11) and (5.12) that

$$
\mathrm{d} \ln \mathrm{V}(x(\mathrm{t}), \mathrm{r}(\mathrm{t})) \geqslant\left[\pi_{\mathrm{II}}(1-\bar{\mu})+\hat{\mathrm{a}}(\mathrm{I})-\frac{\hat{\lambda}}{2 \hat{\mathrm{c}}(\mathrm{I})}|x|\right] \mathrm{dt} .
$$


Hence

$$
\frac{\ln V(x(t), r(t))}{t}+\frac{1}{t} \frac{\hat{\lambda}}{2 \hat{c}(I)} \int_{0}^{t}|x(s)| d s \geqslant \frac{\ln V\left(x\left(\tau_{0}\right), r\left(\tau_{0}\right)\right)}{t}+\frac{1}{t} \int_{0}^{t}\left[\pi_{I I}(1-\mu)+\hat{a}(r(s))\right] d s
$$

So we have

$$
\frac{\hat{\lambda}}{2 \hat{c}(I)} \liminf _{t \rightarrow \infty} \frac{1}{t} \int_{0}^{t}|x(s)| d s \geqslant \pi_{I I}(1-\mu)+\liminf _{t \rightarrow \infty} \frac{1}{t} \int_{0}^{t} \hat{a}(r(s)) d s,
$$

which implies the other required assertion (5.8). This completes the proof of Theorem 5.3.

\section{Numerical simulation}

To illustrate our theoretical results developed in the paper, i.e., stochastic permanence and extinction in mean, we present some numerical examples. Let $(r(t))_{t \geqslant 0}$ be a right-continuous Markov chain taking values in $S=\{0,3\} \subset \widetilde{S}=\{0,1,2,3\}$. Here, for convenience, we only discuss two states, i.e.,

$$
\mathrm{S}=\{0,3\} \subset \widetilde{\mathrm{S}}=\{0,1,2,3\} .
$$

As noted in Section 1, we may regard SDE (2.1) as the result of the following two equations:

$$
\left\{\begin{array}{l}
\frac{d x_{1}}{d t}=x_{1}\left[a_{1}(0)-b_{1}(0) x_{1}-g_{1}(0) x_{2}\right] \\
\frac{d x_{2}}{d t}=x_{2}\left[a_{2}(0)-b_{2}(0) x_{2}-g_{2}(0) x_{1}\right] \\
\frac{d y}{d t}=y\left[a_{3}(0)-b_{3}(0) y\right]
\end{array}\right.
$$

and

$$
\left\{\begin{array}{l}
\frac{d x_{1}}{d t}=x_{1}\left[a_{1}(3)-b_{1}(3) x_{1}-d_{1}(3) y-g_{1}(3) x_{2}\right] \\
\frac{d x_{2}}{d t}=x_{2}\left[a_{2}(3)-b_{2}(3) x_{2}-d_{2}(3) y-g_{2}(3) x_{1}\right] \\
\frac{d y}{d t}=y\left[a_{3}(3)-b_{3}(3) y+e_{1}(3) x_{1}+e_{2}(3) x_{2}\right],
\end{array}\right.
$$

switching from one population to the other per the behaviour of the Markovian chain $r(t)$. Here, subsystem (6.1) implies that the predator $y$ will not capture the species $x_{i}(i=1,2)$, but two different prey compete with each other. In subsystem (6.2), we assume that both interactions, i.e., prey and competition will occur. This means that predator and prey can meet together, including the two competition species of prey.

Table 1: Parameters of the subsystems (6.1) and (6.2).

\begin{tabular}{lcccccc}
\hline Subs. & $\mathrm{r}(\mathrm{t})$ & $\mathrm{a}_{1}(\mathrm{r}(\mathrm{t}))$ & $\mathrm{b}_{1}(\mathrm{r}(\mathrm{t}))$ & $\mathrm{d}_{1}(\mathrm{r}(\mathrm{t}))$ & $\mathrm{g}_{1}(\mathrm{r}(\mathrm{t}))$ & $\mathrm{a}_{2}(\mathrm{r}(\mathrm{t}))$ \\
\hline$(6.1)$ & 0 & 0.8 & 0.3 & 0 & 0.2 & 1.3 \\
$(6.2)$ & 3 & 1.2 & 0.4 & 0.35 & 0.2 & 1.1 \\
\hline $\mathrm{b}_{2}(\mathrm{r}(\mathrm{t}))$ & $\mathrm{d}_{2}(\mathrm{r}(\mathrm{t}))$ & $\mathrm{g}_{2}(\mathrm{r}(\mathrm{t}))$ & $\mathrm{a}_{3}(\mathrm{r}(\mathrm{t}))$ & $\mathrm{b}_{3}(\mathrm{r}(\mathrm{t}))$ & $\left.\mathrm{e}_{1}(\mathrm{r}(\mathrm{t}))\right)$ & $\mathrm{e}_{2}(\mathrm{r}(\mathrm{t}))$ \\
\hline 0.3 & 0 & 0.1 & 1.2 & 0.6 & 0 & 0 \\
0.6 & 0.32 & 0.09 & -0.3 & 0.1 & 0.3 & 0.2 \\
\hline
\end{tabular}


Table 2: Parameters of the subsystems (6.1) and (6.2).

\begin{tabular}{lcccccc}
\hline Subs. & $\mathrm{r}(\mathrm{t})$ & $\mathrm{a}_{1}(\mathrm{r}(\mathrm{t}))$ & $\mathrm{b}_{1}(\mathrm{r}(\mathrm{t}))$ & $\mathrm{d}_{1}(\mathrm{r}(\mathrm{t}))$ & $\mathrm{g}_{1}(\mathrm{r}(\mathrm{t}))$ & $\mathrm{a}_{2}(\mathrm{r}(\mathrm{t}))$ \\
\hline$(6.1)$ & 0 & -0.1 & 0.3 & 0 & 0.2 & -0.7 \\
$(6.2)$ & 3 & -0.7 & 0.4 & 0.25 & 0.13 & -1 \\
\hline $\mathrm{b}_{2}(\mathrm{r}(\mathrm{t}))$ & $\mathrm{d}_{2}(\mathrm{r}(\mathrm{t}))$ & $\mathrm{g}_{2}(\mathrm{r}(\mathrm{t}))$ & $\mathrm{a}_{3}(\mathrm{r}(\mathrm{t}))$ & $\mathrm{b}_{3}(\mathrm{r}(\mathrm{t}))$ & $\left.\mathrm{e}_{1}(\mathrm{r}(\mathrm{t}))\right)$ & $\mathrm{e}_{2}(\mathrm{r}(\mathrm{t}))$ \\
\hline 0.3 & 0 & 0.2 & -0.1 & 0.4 & 0 & 0 \\
0.6 & 0.12 & 0.18 & -0.8 & 0.1 & 0.15 & 0.2 \\
\hline
\end{tabular}

Let $\theta=0.2, \alpha=0.09, \bar{C}=I \in R^{2 \times 2}, q(I)=(1,1)^{\top}, \Pi=\left(\begin{array}{cc}-2 & 2 \\ 5.6 & -5.6\end{array}\right)$ and $P=\left(\begin{array}{cc}0.8 & 0.2 \\ 0.56 & 0.44\end{array}\right)$. We can easily determine from Table 1

$$
A_{11}>0\left(A_{31}>0\right), A_{11} A_{12}-A_{13}>0\left(A_{31} A_{32}-A_{33}>0\right), \text { and } A_{13}>0\left(A_{13}>0\right),
$$

thus (3.1) holds. Meanwhile,

$$
q(0) \theta \hat{a}(0)-\alpha q(0)=-0.01<0,
$$

and

$$
q(3) \theta \hat{a}(3)-\alpha q(3)=-0.15<0,
$$

may also be determined. All conditions of Theorem 4.3 hold, as shown in Figure 1 (a), SDE (2.1) is stochastically permanent. Moreover, we take another parameter shown in Table 2 and let $\Pi=\left(\begin{array}{cc}-2 & 2 \\ 5.6 & -4.4\end{array}\right)$ and $\mathrm{P}=\left(\begin{array}{cc}0.8 & 0.2 \\ 0.56 & 0.44\end{array}\right)$. We can compute $\mu=1$ and find that (6.3) holds, we omit the validation here, as the same holds true for the following examples. Moreover,

$$
\begin{aligned}
& \pi_{00}(1-\mu)+\limsup _{t \rightarrow \infty} \frac{1}{t} \int_{\tau_{0}}^{t} \check{a}(0)=-0.1<0, \\
& \pi_{33}(1-\mu)+\limsup _{t \rightarrow \infty} \frac{1}{t} \int_{\tau_{0}}^{t} \check{a}(3)=-0.7<0 .
\end{aligned}
$$

All assumptions in Theorem 4.4 hold. From the numerical simulation in Figure 1 (b), we can see that SDE (2.1) becomes extinct.

The two numerical simulations demonstrate our results. To further explore the additional dynamic properties of SDE (2.1) under Markovian switching, we consider the following examples in Tables 3-6.

Table 3: Parameters of the subsystems (6.1) and (6.2).

\begin{tabular}{lccccccc}
\hline Subs. & $\mathrm{r}(\mathrm{t})$ & $\mathrm{a}_{1}(\mathrm{r}(\mathrm{t}))$ & $\mathrm{b}_{1}(\mathrm{r}(\mathrm{t}))$ & $\mathrm{d}_{1}(\mathrm{r}(\mathrm{t}))$ & $\mathrm{g}_{1}(\mathrm{r}(\mathrm{t}))$ & $\mathrm{a}_{2}(\mathrm{r}(\mathrm{t}))$ & $\mathrm{b}_{2}(\mathrm{r}(\mathrm{t}))$ \\
\hline$(6.1)$ & 0 & -1.1 & 0.3 & 0 & 0.2 & -1.6 & 0.3 \\
$(6.2)$ & 3 & 3 & 0.4 & 0.35 & 0.13 & 2.8 & 0.6 \\
\hline $\mathrm{d}_{2}(\mathrm{r}(\mathrm{t}))$ & $\mathrm{g}_{2}(\mathrm{r}(\mathrm{t}))$ & $\mathrm{a}_{3}(\mathrm{r}(\mathrm{t}))$ & $\mathrm{b}_{3}(\mathrm{r}(\mathrm{t}))$ & $\mathrm{e}_{1}(\mathrm{r}(\mathrm{t}))$ & $\mathrm{e}_{2}(\mathrm{r}(\mathrm{t}))$ & $\chi$ & Fig \\
\hline 0 & 0.1 & -0.4 & 0.3 & 0 & 0 & Extinct & Figure 2 (a) \\
0.32 & 0.2 & -0.1 & 0.1 & 0.3 & 0.2 & Permanent & Figure 2 (b) \\
\hline
\end{tabular}

Furthermore, in Table 3, if we keep all parameters unchanged and just adjust the values of transition (or generator) between state 0 (extinctive state) and 3 (permanent state), then from simulations in Figure 2, we can see that the extinction and permanence of SDE (2.1) are significantly changed. The details are provided in Table 4. 
Table 4: Values of the generator and transition rate.

\begin{tabular}{lcccc}
\hline case & $\Pi$ & $\mathrm{P}$ & $\chi$ & Fig \\
\hline 1 & $\left(\begin{array}{cc}-3 & 3 \\
8 & -8\end{array}\right)$ & $\left(\begin{array}{ll}0.7 & 0.3 \\
0.8 & 0.2\end{array}\right)$ & Extinct & Figure 2 (c) \\
2 & $\left(\begin{array}{cc}-3.5 & 3.5 \\
5.5 & -5.5\end{array}\right)$ & $\left(\begin{array}{ll}0.65 & 0.35 \\
0.55 & 0.45\end{array}\right)$ & Extinct & Figure 2 (d) \\
3 & $\left(\begin{array}{cc}-4 & 4 \\
5 & -5\end{array}\right)$ & $\left(\begin{array}{ll}0.6 & 0.4 \\
0.5 & 0.5\end{array}\right)$ & Extinct & Figure 2 (e) \\
4 & $\left(\begin{array}{cc}-5.5 & 5.5 \\
4 & -4\end{array}\right)$ & $\left(\begin{array}{ccc}0.45 & 0.55 \\
0.4 & 0.6\end{array}\right)$ & Permanent & Figure 2 (f) \\
\hline
\end{tabular}

Table 5: Parameters of the subsystems (6.1) and (6.2).

\begin{tabular}{lccccccc}
\hline Subs. & $\mathrm{a}_{1}(\mathrm{r}(\mathrm{t}))$ & $\mathrm{b}_{1}(\mathrm{r}(\mathrm{t}))$ & $\mathrm{d}_{1}(\mathrm{r}(\mathrm{t}))$ & $\mathrm{g}_{1}(\mathrm{r}(\mathrm{t}))$ & $\mathrm{a}_{2}(\mathrm{r}(\mathrm{t}))$ & $\mathrm{b}_{2}(\mathrm{r}(\mathrm{t}))$ & $\mathrm{d}_{2}(\mathrm{r}(\mathrm{t}))$ \\
\hline$(6.1)$ & 1.4 & 0.5 & 0 & 0.5 & 1.5 & 0.4 & 0 \\
$(6.2)$ & 3 & 0.4 & 0.35 & 0.15 & 2.8 & 0.6 & 0.32 \\
\hline $\mathrm{g}_{2}(\mathrm{r}(\mathrm{t}))$ & $\mathrm{a}_{3}(\mathrm{r}(\mathrm{t}))$ & $\mathrm{b}_{3}(\mathrm{r}(\mathrm{t}))$ & $\mathrm{e}_{1}(\mathrm{r}(\mathrm{t}))$ & $\mathrm{e}_{2}(\mathrm{r}(\mathrm{t}))$ & $\mathrm{x}_{1}$ & $\mathrm{x}_{2}$ & Fig \\
\hline 0.3 & 0.4 & 0.3 & 0 & 0 & Extinct & Permanent & Figure 3 (a) \\
0.5 & -0.1 & 0.1 & 0.2 & 0.2 & Permanent & Extinct & Figure 3 (b) \\
\hline
\end{tabular}

Table 6: Values of the generator and transition rate.

\begin{tabular}{|c|c|c|c|c|c|}
\hline case & $\Pi$ & $P$ & $x$ & Fig & \\
\hline 1 & $\left.\begin{array}{c}2 \\
-7\end{array}\right)$ & $\left(\begin{array}{ll}0.8 & 0.2 \\
0.7 & 0.3\end{array}\right)$ & Extinct & Permanent & Figure $3(\mathrm{c})$ \\
\hline 2 & $\left(\begin{array}{cc}-2.5 & 2.5 \\
5.5 & -5.5\end{array}\right)$ & $\left(\begin{array}{ll}0.75 & 0.25 \\
0.55 & 0.45\end{array}\right)$ & Extinct & Permanent & Figure $3(d)$ \\
\hline 3 & & $\left(\begin{array}{ll}0.5 & 0.5 \\
0.5 & 0.5\end{array}\right)$ & Permanent & Permanent & Figure $3(\mathrm{e})$ \\
\hline 4 & $\left(\begin{array}{cc}-6.5 & 6.5 \\
3 & -3\end{array}\right)$ & $\left(\begin{array}{cc}0.35 & 0.65 \\
0.3 & 0.7\end{array}\right)$ & Permanent & Extinct & Figure $3(\mathrm{f})$ \\
\hline
\end{tabular}

Moreover, if we consider the influence of the different transition rates between states 0 and 3 on competitors $x_{1}$ and $x_{2}$, such as the parameters used in Table 5 under different transition rates in Table 6, we can see that the system (2.1) displays complicated phenomena, i.e., $x_{2}$ excluding $x_{1}$, coexistence of two competitors and $x_{1}$ excluding $x_{2}$.

(a)

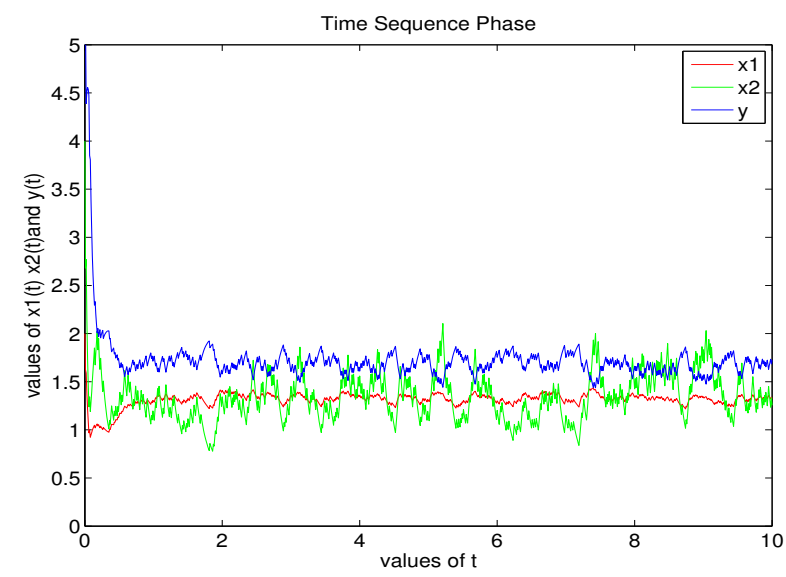

(b)

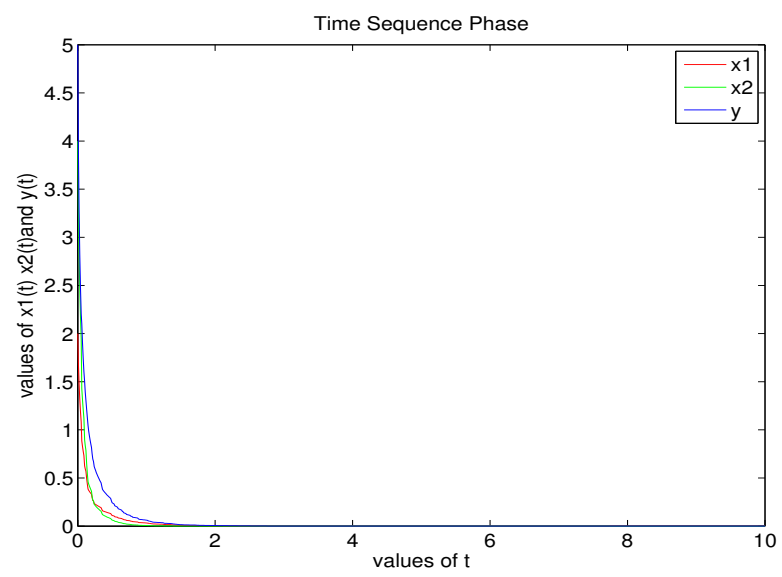

Figure 1: The dynamical behavior of the SDE (2.1). Here, we take the initial value $x_{0}=\left(x_{10}, x_{20}, y_{0}\right)=(2,4,5)$ 
(a)

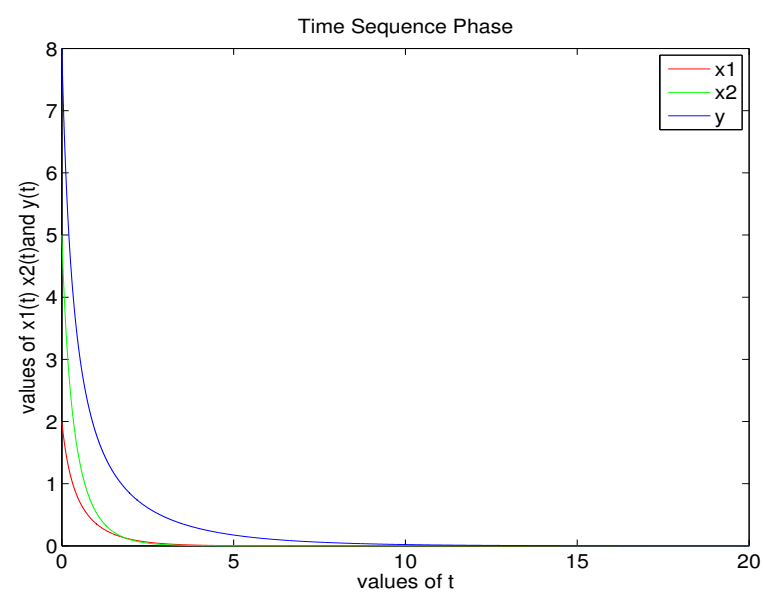

(c)

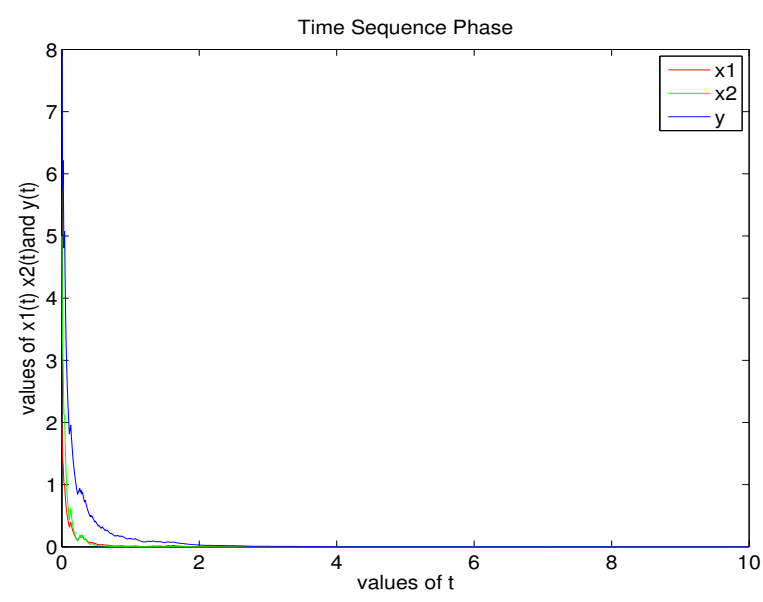

(e)

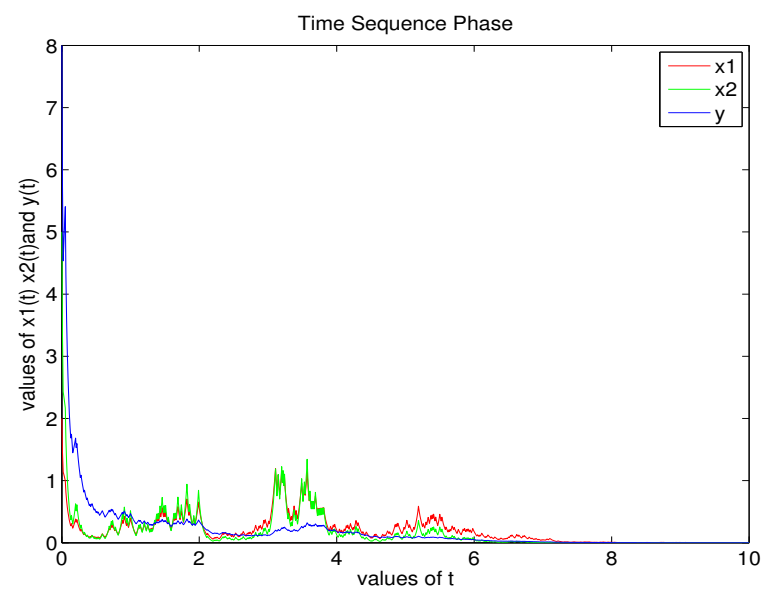

(b)

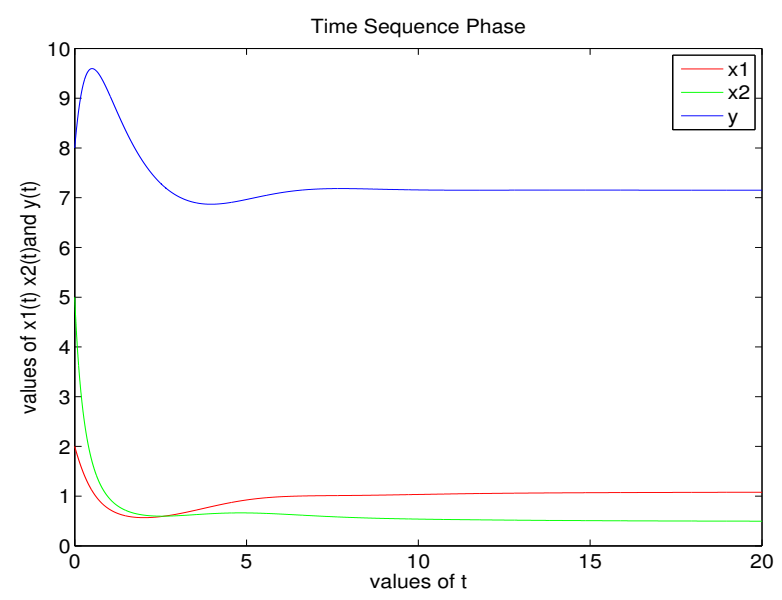

(d)

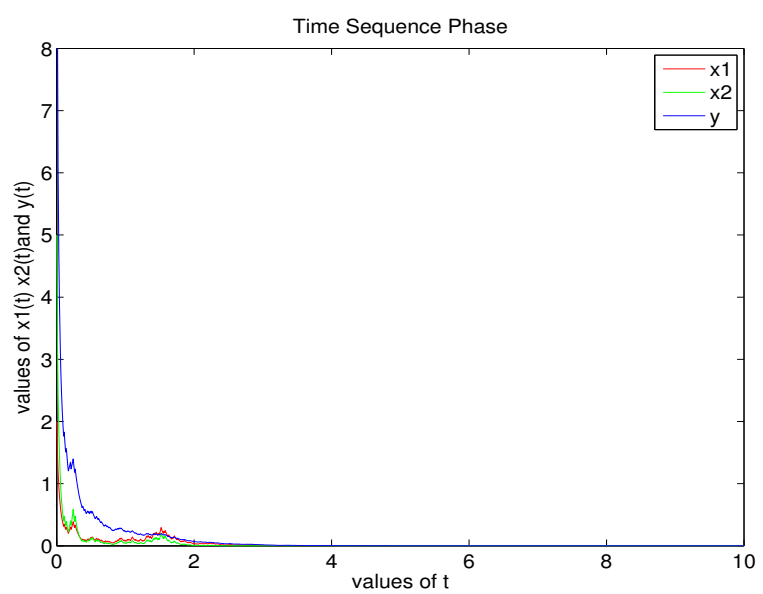

(f)

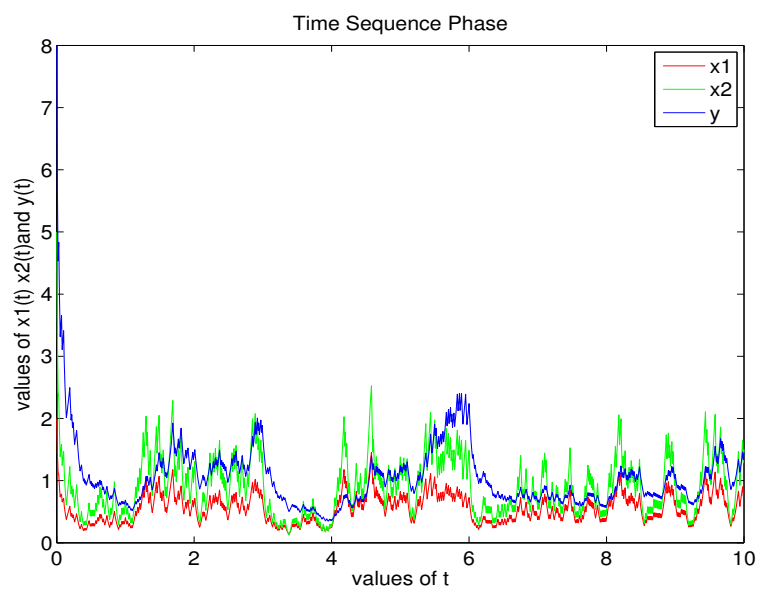

Figure 2: (a,b): The dynamical behavior of deterministic subsystems (6.1) and (6.2) respectively. (c,d,e,f): The dynamical behavior of the SDE (2.1). Here, we take the initial value $x_{0}=\left(x_{10}, x_{20}, y_{0}\right)=(2,5,8)$. 
(a)

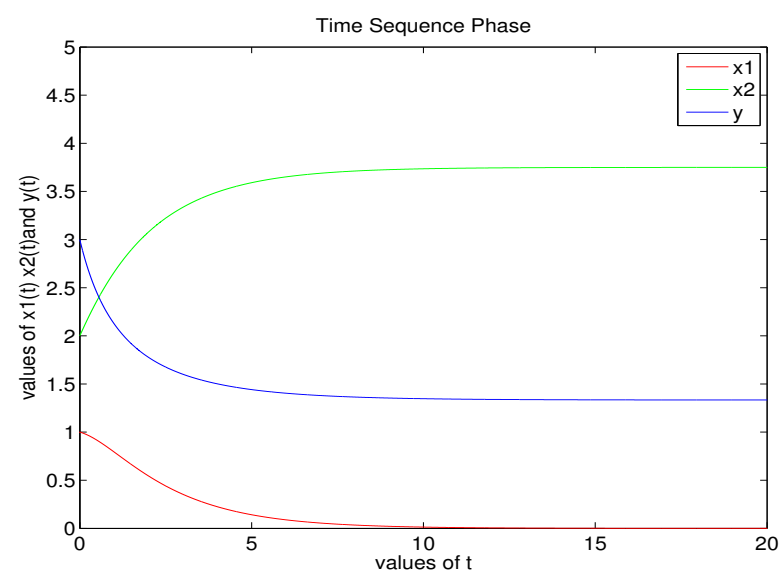

(c)

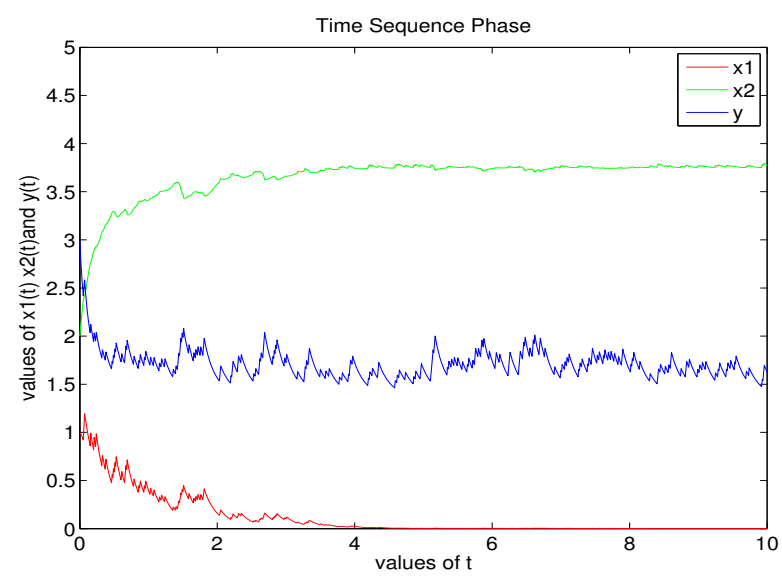

(e)

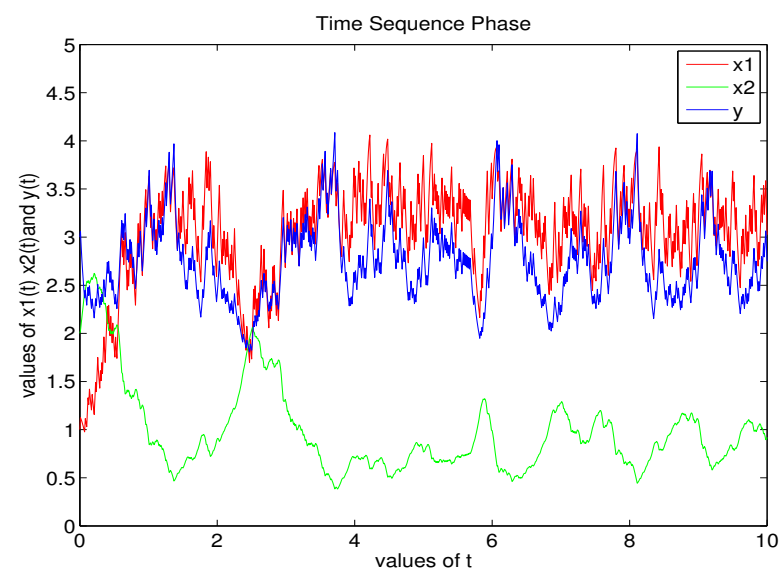

(b)

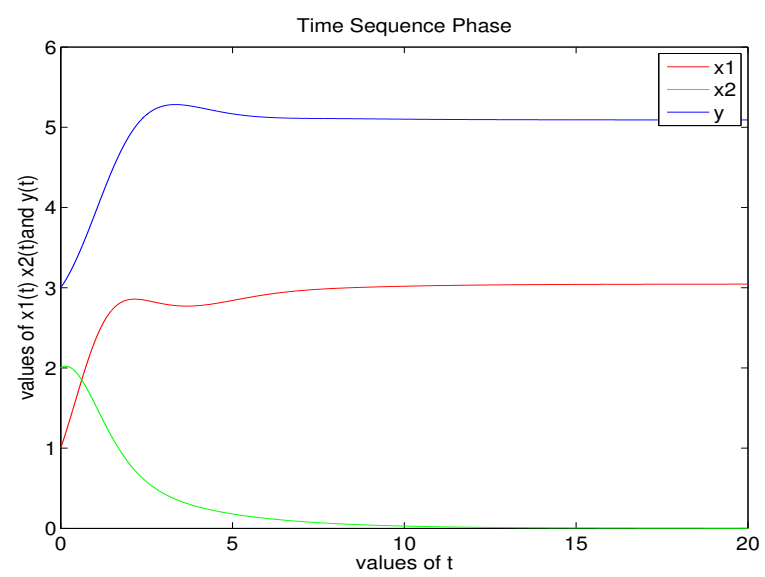

(d)

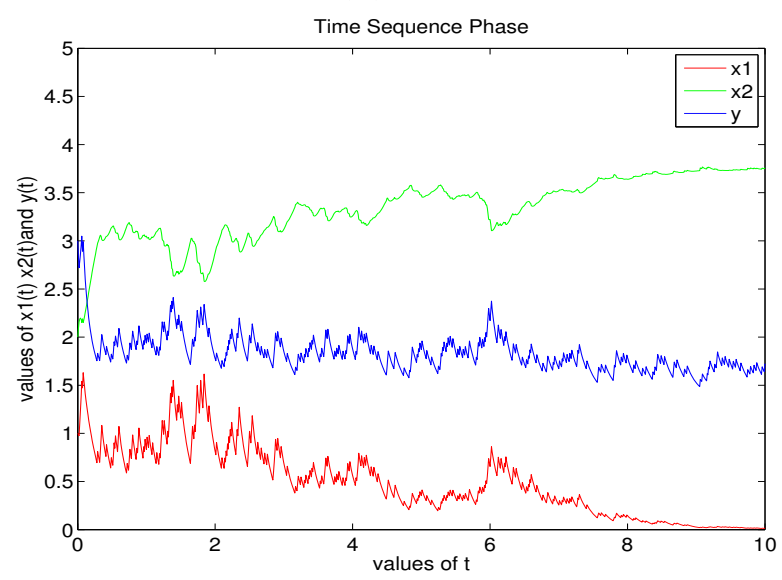

(f)

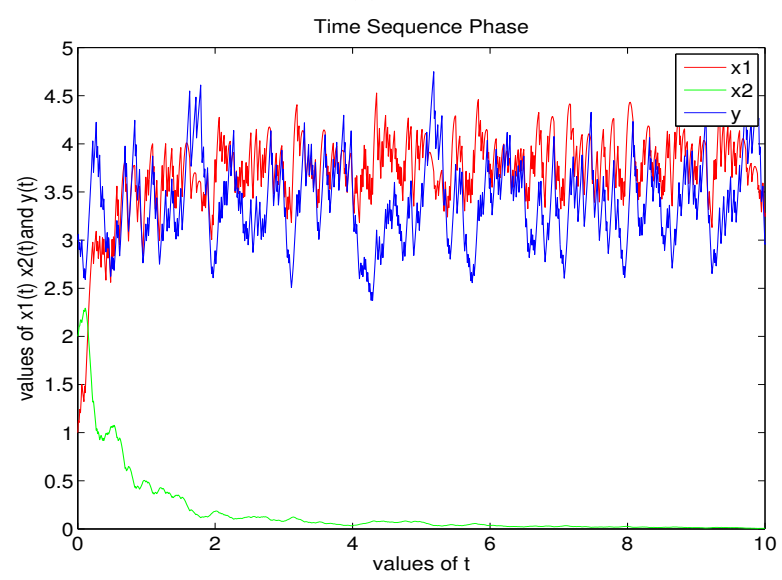

Figure 3: $(\mathrm{a}, \mathrm{b})$ : The dynamical behavior of deterministic systems (6.1) and (6.2), respectively. (c,d,e,f): The dynamical behavior of the SDE (2.1). Here, we take the initial value $x_{0}=\left(x_{10}, x_{20}, y_{0}\right)=(1,2,3)$.

\section{Discussion}

In this paper, we have established a predator-prey system that includes competition between prey and stochastic interactions between predator and multiple prey under Markovian switching. Theorem 4.3 
tells us that if every subsystem of the SDE (2.1) is permanent, the overall behavior, i.e., SDE (2.1) remains stochastically permanent. On the other hand, if $\pi_{\mathrm{II}}(1-\mu)+\limsup _{\mathrm{t} \rightarrow \infty} \frac{1}{\mathrm{t}} \int_{0}^{\mathrm{t}} \check{\mathrm{a}}(\mathrm{I}) \mathrm{ds}<0$ for some I $\in \tilde{\mathrm{S}}$, then subsystem of SDE (2.1) on state I is extinct. Hence, Theorem 4.4 tells us that if every subsystem of SDE (2.1) is extinct, the overall behaviour of system (2.1) is extinct. Theorems 4.3 and 4.4 also tell us that some subsystems in SDE (2.1) are permanent, while others are extinct, as shown in Table 4, the overall behaviour may be stochastically permanent or extinct. When we further increase the transition rates from the extinct state ( 0 state) to the permanent state ( 3 state) and from the permanent state ( 3 state) to the permanent state ( 3 state), i.e., $p_{03}$ and $p_{33}$, the overall behavior of SDE (2.1) will be stochastically permanent if the transition rates $p_{03}$ and $p_{33}$ are greater enough than $p_{00}$ and $p_{30}$.

Furthermore, Markovian switching also imposes constraints on competitors. In Table 6, with the possibility of increasing the survival (competitive force) of species $x_{1}$, some interesting phenomena occur: species $x_{2}$ excludes $x_{1}$ (Figure 3 (c)-(d)), the coexistence of two competitors (Figure 3 (e)) and $x_{1}$ excludes $x_{2}$ (Figure $3(\mathrm{f})$ ). This means that one species may tend to eliminate another species in one set of environmental conditions, but the reverse may occur in a different set of environmental conditions, with the result that the two species may oscillate in density as the environment fluctuates. If inferior species can adjust themselves to offset the fatal impacts from nature, they can coexist with superior competition and even exclusively survive. The results obtained in this paper are different from those obtained with solely deterministic models, which assume the consistency of ecological environments and ignore so many undeterminable factors that occur stochastically in real ecosystems. This can greatly impact on the balance of species in common habitats.

Briefly, a stochastic predator-prey model that includes prey who are competing is a more meaningful model. Due to a shortage of analytical techniques on the stochastic model, the threshold value between the rate of permanence and extinction has not been studied in the present paper. Additionally, the open question of how to guarantee the coexistence of species remains. Thus, great efforts should be devoted to find these answers. We will study this problem in our future work.

\section{Appendix A. Proof of Theorem 3.1}

Proof. It is easy to verify that the coefficients of the model (2.1) are satisfied the local Lipschitz condition in $x(t)$, then there is a unique maximal local solution $x(r(t))$ on $\left[\tau_{0}^{+}, \tau_{e}\right)$, where $\tau_{e}$ is explosion time. To show this solution is global, we need to show that $\tau_{e}=\infty$ a.s.. Let $m_{0}>0$ be sufficient large for every component $x\left(\tau_{0}\right)$ lying within the interval $\left[\frac{1}{m_{0}}, m_{0}\right]$. For each integer $m \geqslant m_{0}$, define the stop time

$$
\tau_{m}=\inf \left\{t \in\left[0, \tau_{e}\right): \min \left\{x_{1}, x_{2}, y\right\} \leqslant \frac{1}{m_{0}} \text { or } \max \left\{x_{1}, x_{2}, y\right\} \geqslant m_{0}\right\},
$$

where throughout this paper we set $\inf \emptyset=\infty$ (as usual $\emptyset$ denotes the empty set). Clearly, $\tau_{m}$ is increasing as $m \rightarrow \infty$. Set $\tau_{\infty}=\lim _{t \rightarrow \infty} \tau_{m}$, whence $\tau_{\infty}<\tau_{e}$ a.s.. If we can show that $\tau_{\infty}=\infty$ a.s., then $\tau_{e}=\infty$ a.s.. If this statement is false, there is a pair of constants $T>0$ and $\epsilon \in(0,1)$ such that

$$
P\left\{\tau_{\infty} \leqslant T\right\}>\epsilon .
$$

Hence there is an integer $m_{1} \geqslant m_{0}$ such that

$$
P\left\{\tau_{m} \leqslant T\right\}>\epsilon, \quad \forall m \geqslant m_{1} .
$$

Define a $C^{2}$-function $V: R_{+}^{n} \times \tilde{S} \rightarrow R_{+}$by

$$
\mathrm{V}(\mathrm{x}, \mathrm{I})=\mathrm{c}_{1}(\mathrm{I})\left(\mathrm{x}_{1}-1-\ln \mathrm{x}_{1}\right)+\mathrm{c}_{2}(\mathrm{I})\left(\mathrm{x}_{2}-1-\ln \mathrm{x}_{2}\right)+\mathrm{c}_{3}(\mathrm{I})(\mathrm{y}-1-\ln \mathrm{y}) .
$$

The nonnegativity of this function can be seen from

$$
u-1-\ln u \geqslant 0 \text { on } u>0 .
$$


If $x(t) \in R_{+}^{3}$, we have

$$
\begin{aligned}
\mathrm{dV}(x, \mathrm{I})= & \mathcal{L} V(x, \mathrm{I}) d t \\
= & \left\{x^{\top} \overline{\mathrm{C}}(\mathrm{I}) \mathrm{a}(\mathrm{I})+x^{\top} \overline{\mathrm{C}}(\mathrm{I}) \mathrm{A}(\mathrm{I}) x-\mathrm{C}(\mathrm{I})[\mathrm{a}(\mathrm{I})+\mathrm{A}(\mathrm{I}) \mathrm{x}]\right. \\
& \left.+\sum_{\mathrm{J}=1}^{4} \pi_{\mathrm{IJ}} \mathrm{V}(\mathrm{x}, \mathrm{J})\right\} \mathrm{dt} \\
= & \left\{x^{\top} \overline{\mathrm{C}}(\mathrm{I}) \mathrm{a}(\mathrm{I})-\mathrm{C}(\mathrm{I}) \mathrm{A}(\mathrm{I}) x+\frac{x^{\top}\left(\overline{\mathrm{C}}(\mathrm{I}) \mathrm{A}(\mathrm{I})+\mathrm{A}(\mathrm{I})^{\top} \overline{\mathrm{C}}(\mathrm{I})\right) x}{2}-\mathrm{C}(\mathrm{I}) \mathrm{a}(\mathrm{I})\right. \\
& \left.+\sum_{J=1}^{4} \pi_{\mathrm{IJ}} \mathrm{V}(x, \mathrm{~J})\right\} \mathrm{dt} \\
\leqslant & \left\{-\frac{\lambda}{2}|x|^{2}+x^{\top} \overline{\mathrm{C}}(\mathrm{I}) \mathrm{a}(\mathrm{I})-\mathrm{C}(\mathrm{I}) \mathrm{A}(\mathrm{I}) x-\mathrm{C}(\mathrm{I}) \mathrm{a}(\mathrm{I})+\sum_{\mathrm{J}=1}^{4} \pi_{\mathrm{IJ}} \mathrm{V}(x, \mathrm{~J})\right\} \mathrm{dt} .
\end{aligned}
$$

Moreover, there is a constant $\mathrm{K}_{3}>0$ such that

$$
\max _{I \in \tilde{S}}\left\{x^{\top} \bar{C}(I) a(I)-C(I) A(I) x-C(I) a(I)\right\} \leqslant K_{3}(1+|x|) .
$$

Substituting this inequality into (A.2) yields

$$
\mathcal{L V}(x, \mathrm{I}) \leqslant \mathrm{K}_{3}(1+|x|)+\sum_{\mathrm{J}=1}^{4} \pi_{\mathrm{IJ}} \mathrm{V}(\mathrm{x}, \mathrm{J}) .
$$

Noticing that $u \leqslant 2(u-1-\ln u)+2$ on $u>0$, we have

$$
\begin{aligned}
|x| \leqslant & x_{1}+x_{2}+y \leqslant\left[2\left(x_{1}-1-\ln x_{1}\right)+2+2\left(x_{2}-1-\ln x_{2}\right)+2\right. \\
& +2(y-1-\ln y)+2] \\
\leqslant & 6+\frac{2}{\hat{c}}\left[c_{1}(I)\left(x_{1}-1-\ln x_{1}\right)+c_{2}(I)\left(x_{2}-1-\ln x_{2}\right)+c_{3}(I)(y-1-\ln y)\right] \\
= & 6+\frac{2}{\hat{c}} V(x, I) .
\end{aligned}
$$

By the definition of $V$, for any $I, J \in \tilde{S}$, we have

$$
\sum_{J=1}^{4} \pi_{I J} V(x, J) \leqslant 4 \pi \mu V(x, I) .
$$

We therefore obtain from (A.3), (A.4) and (A.5) that

$$
\mathcal{L V}(x, \mathrm{I}) \leqslant \mathrm{K}_{4}[1+\mathrm{V}(\mathrm{x}, \mathrm{I})]
$$

where $K_{4}$ is a positive constant. Integrating both sides of the inequality (A.6) from $\tau_{m}^{+}$to $\tau_{m} \wedge T$ and then taking expectations, one has

$$
\mathrm{EV}\left(x\left(\tau_{\mathrm{m}} \wedge \mathrm{T}\right), \mathrm{r}\left(\tau_{\mathrm{m}} \wedge \mathrm{T}\right)\right) \leqslant \mathrm{EV}\left(x\left(\tau_{\mathrm{m}}^{+}\right), \mathrm{r}\left(\tau_{\mathrm{m}}^{+}\right)\right)+\mathrm{E} \int_{\tau_{\mathrm{m}}}^{\tau_{\mathrm{m}} \wedge \mathrm{T}} \mathrm{K}_{4}[1+\mathrm{V}(x, \mathrm{I})] \mathrm{ds}
$$


Hence

$$
\begin{aligned}
\operatorname{EV}\left(x\left(\tau_{m} \wedge T\right), r\left(\tau_{m} \wedge T\right)\right) \leqslant & E V\left(x\left(\tau_{m}\right), r\left(\tau_{m}\right)\right)+K_{4} T+K_{4} E \int_{\tau_{m}}^{\tau_{m} \wedge T} V(x, I) d s, \\
\leqslant & V\left(x\left(\tau_{0}\right), r\left(\tau_{0}\right)\right)+K_{4} T \\
& +K_{4} E \int_{0}^{T} V\left(x\left(\tau_{m} \wedge t\right), I\left(\tau_{m} \wedge t\right)\right) d t .
\end{aligned}
$$

By the Gronwall inequality, we know

$$
\operatorname{EV}\left(x\left(\tau_{m} \wedge T\right), r\left(\tau_{m} \wedge T\right)\right) \leqslant\left[V\left(x\left(\tau_{0}, r\left(\tau_{0}\right)\right)+K_{4} T\right] e^{K_{4} T} .\right.
$$

Set $\Omega_{m}=\left\{\tau_{m} \leqslant T\right\}$ for $m \geqslant m_{1}$ and by (A.1), $P\left(\Omega_{m}\right) \geqslant \epsilon$. Note that for every $\omega \in \Omega_{m}$, we have $x_{i}(i=1,2)$ or $y$ equals either $m$ or $\frac{1}{m}$, and hence $V\left(x\left(\tau_{m}, \omega\right)\right)$ is no less than either $\hat{c}(m-1-\ln m)$ or $\hat{\mathrm{c}}\left(\frac{1}{\mathrm{~m}}-1-\ln \frac{1}{\mathrm{~m}}\right)=\hat{\mathrm{c}}\left(\frac{1}{\mathrm{~m}}-1+\ln \mathrm{m}\right)$. Consequently,

$$
\mathrm{V}\left(x\left(\tau_{\mathrm{m}}, \omega\right), \mathrm{r}\left(\tau_{\mathrm{m}}, \omega\right)\right) \geqslant \hat{c}\left((\mathrm{~m}-1-\ln \mathrm{m}) \wedge\left(\frac{1}{\mathrm{~m}}-1+\ln \mathrm{m}\right)\right)
$$

Hence

$$
\begin{aligned}
{\left[\mathrm{V}\left(x\left(\tau_{0}\right), \mathrm{r}\left(\tau_{0}\right)\right)+\mathrm{K}_{4} \mathrm{~T}\right] \mathrm{e}^{\mathrm{K}_{4} \mathrm{~T}} } & \geqslant \mathrm{E}\left[1_{\Omega_{\mathrm{m}}} \mathrm{V}\left(x\left(\tau_{\mathrm{m}}, \omega\right), \mathrm{r}\left(\tau_{\mathrm{m}}, \omega\right)\right)\right] \\
& \geqslant \epsilon \hat{c}\left((\mathrm{~m}-1-\ln \mathrm{m}) \wedge\left(\frac{1}{\mathrm{~m}}-1+\ln \mathrm{m}\right)\right),
\end{aligned}
$$

where $1_{\Omega_{m}}$ is the indicator function of $\Omega_{m}$. Let $m \rightarrow \infty$ which leads to the contradiction

$$
\infty>\left[\mathrm{V}\left(x\left(\tau_{0}\right), \mathrm{r}\left(\tau_{0}\right)\right)+\mathrm{K}_{4} \mathrm{~T}\right] e^{\mathrm{K}_{4} \mathrm{~T}}=\infty .
$$

So we must have $\tau_{\infty}=\infty$ a.s.. The proof of Theorem 3.1 is complete.

\section{Acknowledgment}

This work was supported by The National Natural Science Foundation of P. R. China (11361059, 11401569, 11702237), The Key Project of Laboratory of Xinjiang Province of China (2016D03022), The NSF of Jiangsu Province of China (BK20130779), and The Doctoral Scientific Research Foundation of Xinjiang University (BS160204).

\section{References}

[1] R. Arditi, L. R. Ginzburg, Coupling in predator-prey dynamics: ratio-dependence, J. Theor. Biol., 139 (1989), 311-326. 1

[2] J. R. Beddington, Mutual interference between parasites or predators and its effect on searching efficiency, J. Anim. Ecol., 44 (1975), 331-340.

[3] D. L. DeAngelis, R. A. Goldstein, R. V. O’Neill, A model for tropic interaction, Ecol., 56 (1975), 881-892. 1

[4] H. I. Freedman, Deterministic mathematical models in population ecology, Monographs and Textbooks in Pure and Applied Mathematics, Marcel Dekker, Inc., New York, (1980). 1

[5] H. I. Freedman, S. G. Ruan, Uniform persistence in functional-differential equations, J. Differential Equations, 115 (1995), 173-192. 1

[6] P. Ghirardato, On independence for non-additive measures, with a Fubini theorem, J. Econom. Theory, 73 (1997), 261291. 2.5

[7] M. E. Gilpin, Group selection in predator-prey communities, Princeton Univ. Press, New Jersey, (1975). 1

[8] J. Hofbauer, K. Sigmund, Evolutionary games and population dynamics, Cambridge University Press, Cambridge, (1998). 1

[9] C. S. Holling, The functional response of predators to prey density and its role in mimicry and population regulation, Mem. Ent. Soc. Canada, 46 (1965), 5-60. 1 
[10] S. B. Hsu, T. W. Huang, Global stability for a class of predator-prey systems, SIAM J. Appl. Math., 55 (1955), $763-783$. 1

[11] G. E. Hutchinson, The paradox of the plankton, Am. Nat., 95 (1961), 137-145. 1

[12] X.-Y. Li, D.-Q. Jiang, X.-R. Mao, Population dynamical behavior of Lotka-Volterra system under regime switching, J. Comput. Appl. Math., 232 (2009), 427-448. 1

[13] F.-F. Li, J.-T. Sun, Stability analysis of a reduced model of the lac operon under impulsive and switching control, Nonlinear Anal. Real World Appl., 12 (2011), 1264-1277. 1

[14] D. Liberzon, A. S. Morse, Basic problems in stability and design of switched systems, IEEE Control Syst., 19 (1999), 59-70.

[15] Z.-J. Liu, S.-L. Guo, R.-H. Tan, M. Liu, Modeling and analysis of a non-autonomous single-species model with impulsive and random perturbations, Appl. Math. Model., 40 (2016), 5510-5531. 1

[16] M. Liu, K. Wang, Asymptotic properties and simulations of a stochastic logistic model under regime switching, Math. Comput. Modelling, 54 (2011), 2139-2154. 1

[17] M. Liu, K. Wang, On a stochastic logistic equation with impulsive perturbations, Comput. Math. Appl., 63 (2012), 871-886. 1

[18] G.-R. Liu, J.-R. Yan, Existence of positive periodic solutions for neutral delay Gause-type predator-prey system, Appl. Math. Model., 35 (2011), 5741-5750. 1

[19] X.-R. Mao, C.-G. Yuan, Stochastic differential equations with Markovian switching, Imperial College Press, London, (2006). 1, 2.3, 2.4, 2.6

[20] R. M. May, Stability and complexity in model ecosystems, Princeton University Press, New Jersey, (2001). 1

[21] D. Neal, Introduction to population biology, Cambridge University Press, New York, (2004). 1, 2.1

[22] R. M. Nisbet, W. Gurney, Modelling fluctuating populations, John Wiley and Sons, Chichester and New York, Reprinted in 2003 by Blackburn Press, New Jersey, (1982). 1

[23] J. D. Parrish, S. B. Saila, Interspecific competition, predation and species diversity, J. Theor. Biol., 27 (1970), 207-220. 1

[24] L. L. Rockwood, Introduction to population ecology, John Wiley \& Sons, India, (2015). 1

[25] H.-B. Shi, Y. Li, Global asymptotic stability of a diffusive predator-prey model with ratio-dependent functional response, Appl. Math. Comput., 250 (2015), 71-77. 1

[26] Z.-D. Sun, S. S. Ge, Analysis and synthesis of switched linear control systems, Automatica J. IFAC, 41 (2005), 181-195. 2.2

[27] Y. Takeuchi, Global dynamical properties of Lotka-Volterra systems, World Scientific Publishing Co., Inc., River Edge, NJ, (1996) 1

[28] Y. Takeuchi, N. H. Du, N. T. Hieu, K. Sato, Evolution of predator-prey systems described by a Lotka-Volterra equation under random environment, J. Math. Anal. Appl., 323 (2006), 938-957. 1, 1

[29] A. Vlasic, Stochastic replicator dynamics subject to Markovian switching, J. Math. Anal. Appl., 427 (2015), 235-247. 1

[30] Q. Wang, X.-Z. Liu, Stability criteria of a class of nonlinear impulsive switching systems with time-varying delays, J. Franklin Inst., 349 (2012), 1030-1047. 1

[31] Y.-J. Wang, X.-M. Shi, Z.-Q. Zuo, M. Z. Q. Chen, Y.-T. Shao, On finite-time stability for nonlinear impulsive switched systems, Nonlinear Anal. Real World Appl., 14 (2013), 807-814.

[32] L.-G. Wu, P. Shi, H.-J. Gao, State estimation and sliding-mode control of Markovian jump singular systems, IEEE Trans. Automat. Control, 55 (2010), 1213-1219. 1

[33] A. Yagi, T. V. Ton, Dynamic of a stochastic predator-prey population, Appl. Math. Comput., 218 (2011), 3100-3109. 1

[34] M. Yang, Y.-W. Wang, J.-W. Xiao, Y.-H. Huang, Robust synchronization of singular complex switched networks with parametric uncertainties and unknown coupling topologies via impulsive control, Commun. Nonlinear Sci. Numer. Simul., 17 (2012), 4404-4416. 1

[35] L. Zhang, Z.-D. Teng, The dynamical behavior of a predator-prey system with Gompertz growth function and impulsive dispersal of prey between two patches, Math. Methods Appl. Sci., 39 (2016), 3623-3639. 1

[36] L. Zhang, Z.-D. Teng, Z.-J. Liu, Survival analysis for a periodic predatory-prey model with prey impulsively unilateral diffusion in two patches, Appl. Math. Model., 35 (2011), 4243-4256. 1

[37] L. Zu, D.-Q. Jiang, D. O'Regan, Conditions for persistence and ergodicity of a stochastic Lotka-Volterra predator-prey model with regime switching, Commun. Nonlinear Sci. Numer. Simul., 29 (2015), 1-11. 1 